\title{
NOTES ON THE POISSON FORMULA
}

\author{
A. N. PARSHIN
}

\begin{abstract}
This is a survey of applications of harmonic analysis to the study of the zeta-functions of one-dimensional schemes. A new version of the Tate-Iwasawa method is suggested that involves holomorphic duality for discrete groups instead of Pontryagin duality. A relationship is found between the Poisson formula and the residue formula on the compactification of the holomorphically dual group. Links to explicit formulas for zeta-functions of algebraic curves are found. A numerical analog of these constructions is considered in the appendix written by I. S. Rezvyakova.
\end{abstract}

\section{Contents}

$\S 1 . \quad$ Introduction

$\S 2$. The Tate-Iwasawa method for algebraic curves

2.1. Preliminaries and notation

2.2. Duality and the Fourier transform

2.3. The Weil problem

2.4. Regularization

2.5. Applications

$\S 3 . \quad$ Discrete versions and holomorphic tori $\quad 793$

3.1. Analysis on discrete groups $\quad 793$

3.2. Extended group of divisors $\quad 796$

3.3. Holomorphic tori $\quad 796$

3.4. The Poisson formula and residues $\quad 802$

3.5. Explicit formulas 803

$\S 4$. Trace formula and the Artin representation $\quad 805$

4.1. Zeta functions and étale cohomology 805

4.2. Local Artin representation 807

4.3. Relationship with explicit formulas $\quad 809$

$\S 5$. Number fields (from $\mathbb{C}^{*}$ to $\mathbb{C}$ ) 811

$\S 6$. Appendix (Irina Rezvyakova). Residues of the Dedekind zeta functions of number fields

These notes are a part of my lectures on representations of adelic groups attached to two-dimensional schemes. The main part of the lectures was focused on representation theory for discrete Heisenberg groups and applications to the groups of this type arising from the algebraic surfaces defined over a finite field. The arithmetic surfaces were not considered, except for the remark that the theory for geometric surfaces can be transposed

2010 Mathematics Subject Classification. Primary 11M41.

Key words and phrases. Fourier transform, Poisson formula, zeta-function, holomorphic duality, residues, explicit formulas.

Supported by RFBR (grant no. 11-01-00145-a). 
to this case without changes if we restrict ourselves to the scheme part of the arithmetic surface.

The classical representation theory for discrete groups is not well developed because these groups are mostly not of type I in the von Neumann classification. This implies violation of the main principles of representation theory on Hilbert spaces: the nonuniqueness of a decomposition into irreducible components; bad topology of the unitary dual space; the nonexistence of characters ... (see, e.g., [Dix $)$. By the Thoma theorem, a discrete group is of type I if and only if it has an Abelian subgroup of finite index.

On the other hand, there exists a theory of smooth representations for $p$-adic algebraic groups, which is of principal importance for the Langlands program. Part of this theory is also valid for a more general class of totally disconnected locally compact groups. The discrete groups are a simple particular case of this class of groups, and the general theory delivers a reasonable class of representations, which are representations on a vector space without any topology. The new viewpoint consists in a systematic consideration of purely algebraic induced representations in place of unitary representations on Hilbert spaces.

In the case under consideration, the change of the class of representations will cause the moduli spaces of induced representations to be complex-analytic manifolds. Characters do exist and will then be modular forms. It seems that the more general holomorphic dual space is more adequate for this class of groups than the standard unitary dual going back to the Pontryagin duality for Abelian groups.

This approach can change even the theory of representations for Abelian discrete groups, and its application to the arithmetic of algebraic curves. This point, hopefully, will be clear from these notes.

The lectures were given in both branches of the Steklov Mathematical Institute (St. Petersburg, November 17-20, and Moscow, December 21-30, 2009)1 1 I am very thankful to the audience, especially to Volodya Zhgun, Maxim Korolev and Irina Rezvyakova, for fruitful discussions and to Lawrence Breen who read the final text and made many important remarks. Also, I am grateful to Sergey Gorchinskiǔ and to Igor Zhukov and his students for their help in preparation of these notes. Sergey Gorchinskil also read the final version and made a number of useful remarks.

\section{$\S 1$. INTRODUCTION}

The simplest case of the Poisson summation formula is the following one. Let $\mathbb{R}$ be the additive group of real numbers and $\mathbb{Z}$ the discrete subgroup of integers. Denote by $\mathcal{S}(\mathbb{R})$ the space of smooth functions on $\mathbb{R}$ that decay rapidly together with all their derivatives (= Schwartz functions $[\mathrm{Sch}]$ ). We have the Fourier transform

$$
\mathrm{F}: \mathcal{S}(\mathbb{R}) \rightarrow \mathcal{S}(\mathbb{R})
$$

where

$$
\mathrm{F}(f)(y)=\int_{\mathbb{R}} \exp (2 \pi i x y) f(x) d x
$$

for $f \in \mathcal{S}(\mathbb{R})$ and $y \in \mathbb{R}$. The Poisson formula looks like this (see [GSh):

$$
\sum_{n \in \mathbb{Z}} f(n)=\sum_{n \in \mathbb{Z}}(\mathrm{F} f)(n) .
$$

Here are the main ingredients of this result:

- a locally compact commutative topological group $G$;

- its Pontryagin dual group $\breve{G}$;

${ }^{1} \mathrm{~A}$ substantial part of these notes was also presented in my lectures in the Humboldt University, Berlin, October 2010. 
- a discrete subgroup $\Gamma \subset G$;

- a space of functions $\mathcal{F}(G)$;

- a Haar measure $\mu \in \mu(G)$ on $G$ such that $\mu(G / \Gamma)<\infty$;

- the Fourier transform

$$
\mathrm{F}: \mathcal{F}(G) \rightarrow \mathcal{F}(\check{G}) \text {. }
$$

In the simplest case $G=\mathbb{R}=\breve{G}, \Gamma=\mathbb{Z}, \mu=d x$, and $\mathcal{F}(G)=\mathcal{S}(\mathbb{R})$. In a more general situation,

$$
\sum_{x \in \Gamma} f(x)=\mu(G / \Gamma)^{-1} \sum_{y \in \Gamma^{\perp}}(\mathrm{F} f)(y),
$$

where $\Gamma^{\perp}=\{y \in G:\langle y, x\rangle=1$ for all $x \in \Gamma\}$ is the annihilator (or orthogonal) of $\Gamma$,

$$
\mathrm{F}(f)(y)=\int_{G}\langle y, x\rangle f(x) d \mu(x),
$$

$\langle-,-\rangle: G \times \breve{G} \rightarrow \mathbf{U}(1) \sqrt{2}$ is the canonical pairing of $G$ with $\breve{G}$, and $\mathcal{F}(G)$ is an appropriate space of functions.

Let us outline a proof of the Poisson formula. Take the function $\varphi(g):=\sum_{\gamma \in \Gamma} f(g \gamma)$, $g \in G / \Gamma$ and integrate it over $G / \Gamma$ with characters $\chi \in \breve{G}$ trivial on $\Gamma$ :

$$
(\mathrm{F} \varphi)(\chi)=\int_{G / \Gamma} \chi(g) \varphi(g) d \mu(g) .
$$

The measure on $G / \Gamma$ is basically the same as the original measure on $G$ and the set of characters will be exactly $\Gamma^{\perp}=$ dual to $G / \Gamma$. Thus, the integral is the Fourier transform on the group $G / \Gamma$. Assuming that the integral (in fact, a sum) of this function over $\Gamma^{\perp}$ converges, we have

$$
\sum_{\chi \in \Gamma^{\perp}}(\mathrm{F} \varphi)(\chi)=\varphi(e)=\sum_{\gamma \in \Gamma} f(\gamma)
$$

where the first identity follows from the inversion formula $\mathrm{F} \circ \mathrm{F}=(-1)^{*}$ for the Fourier transform on the group $G / \Gamma$ and its dual group. On the other hand, interchanging summation (defining the $\varphi$ ) and integration in the first integral, we have

$$
\begin{aligned}
(\mathrm{F} \varphi)(\chi) & =\sum_{\gamma \in \Gamma} \int_{G / \Gamma} f(g \gamma) \chi(g) d \mu(g) \\
& =\sum_{\gamma \in \Gamma} \int_{G / \Gamma} f(g \gamma) \chi(g \gamma) d \mu(g)=\int_{G} f(g) \chi(g) d \mu(g)=(\mathrm{F} f)(\chi) .
\end{aligned}
$$

Combining all this, we get the Poisson formula.

Beyond formula (11) and the proof, one can find some functorialities, which will be valid in a more general situation when one has an arbitrary closed subgroup $H \subset G$ instead of the discrete subgroup $\Gamma$. We shall denote by $\mu(G)$ the space of Haar measures on a group $G$. Thus, $\mu(G) \cong \mathbb{C}$, and the Fourier transform is a canonical map

$$
\mathrm{F}_{G}: \mathcal{F}(G) \otimes \mu(G) \rightarrow \mathcal{F}(\check{G})
$$

defined for any locally compact group $G$.

If our group $G$ is totally disconnected, then we set

$$
\mathcal{F}(G)=\mathcal{D}(G)=\{\text { locally constant functions with compact support }\} .
$$

These are the classical spaces introduced by F. Bruhat $[\mathrm{Br}$. For connected Lie groups we can use the space $\mathcal{S}(G)$ of Schwartz functions $\left[\mathrm{Sch}\right.$ and its dual $\mathcal{S}^{\prime}(G)$. The general case reduces easily to these two (see $[\mathrm{Br}]$ ). The Fourier transform for the distribution spaces

\footnotetext{
${ }^{2} \mathbf{U}(1)$ is the group of complex numbers with modulus equal to 1 .
} 
$\mathcal{F}^{\prime}(G)$ such as $\mathcal{D}^{\prime}(G)=\{$ dual to $\mathcal{D}(G)$, i.e., all distributions $\}$ and $\mathcal{S}^{\prime}(G)$ can be defined by duality and will be a map $\mathrm{F}: \mathcal{F}^{\prime}(G) \otimes \mu(G)^{-1} \rightarrow \mathcal{F}^{\prime}(\breve{G})$, where $\mu(G)^{-1}$ is the dual 1-dimensional space. Harmonic analysis includes definitions of the direct and inverse images for functions and distributions in the category of locally compact commutative groups. The Fourier transform will interchange the direct and inverse images.

Suppose we are given a closed subgroup $i: H \rightarrow G$. Denote by $\pi$ the canonical map from $G$ onto $G / H$, by $\alpha$ the embedding of the trivial group $\{e\}$ into $G / H$, and by $\beta$ the surjection of $H^{\perp}$ onto $\{e\}$. Here, $H^{\perp} \subset \check{G}$ is the annihilator of $H$ in $\breve{G}$ defined as above.

Then the diagram

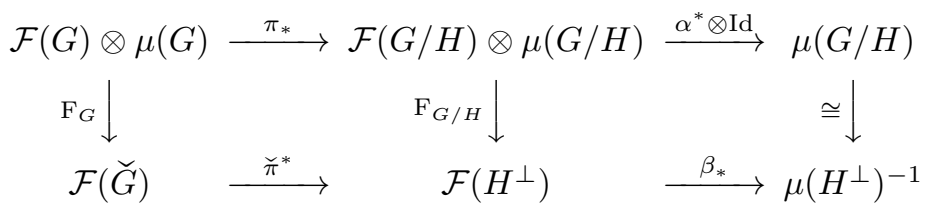

commutes. Here the direct image $\pi_{*}$ is the integral over the fibers of $\pi$ and the inverse image $\pi^{*}$ is restriction to the subgroup $H^{\perp}$. To define the integral, the canonical decomposition $\mu(G)=\mu(H) \otimes \mu(G / H)$ should be used. Since the Haar measure is translation invariant, this gives measures not only on $H=\operatorname{Ker} \pi$, but also on all fibers of $\pi$. The map $\alpha^{*}$ is the evaluation at the point $e$, and $\beta_{*}$ is the integral over $H^{\perp}$. Finally, the canonical isomorphism between measure spaces follows from the relations $(G / H)=H^{\perp}$ and $\mu($ a group $) \otimes \mu($ its dual group $)=\mathbb{C}$.

Then we have the following Poisson formula:

$$
\mathrm{F}\left(\delta_{H, \mu_{0}} \otimes \mu^{-1}\right)=\delta_{H^{\perp}, \mu^{-1} / \mu_{0}^{-1}}
$$

for any closed subgroup $i: H \rightarrow G$. Here

- $\mu_{0} \in \mu(H) \subset \mathcal{D}^{\prime}(H)$;

- $\mu \in \mu(G)$;

- $\delta_{H, \mu_{0}}=i_{*}\left(\mu_{0}\right), \quad i_{*}: \mathcal{D}^{\prime}(H) \rightarrow \mathcal{D}^{\prime}(G)$.

We have used the fact that $\mu\left(H^{\perp}\right)=\mu(G / H)^{-1}=\mu(G)^{-1} \mu(H)$ by the definition of measures.

If the subgroup $H$ is discrete, then for $\mu_{0} \in \mu(H)$ we can take the canonical measure on $H$, the $\delta_{H, \mu_{0}}$ will be exactly the sum over $H$, and we arrive at formula (1). Concerning the general formalism of harmonic analysis over $n$-dimensional local fields and adelic groups $(n=0,1,2)$, we refer to [P1, OP1, OP2]. The basic problem solved there was to extend the classical analysis known for locally compact (and first of all for finite) groups to the case of fields and groups arising from two-dimensional schemes. These fields and groups are no longer locally compact.

Now we briefly outline a principal application of the Poisson formula to investigation of the analytic structure of Riemann's zeta function $\zeta(s)$. We know that

$$
\zeta(s)=\sum_{n \geq 1} n^{-s}=\prod_{p}\left(1-p^{-s}\right)^{-1}=\prod_{p} \zeta_{p}(s),
$$

where $p$ runs through the prime numbers, $s \in \mathbb{C}$, and both expressions are convergent for $\operatorname{Re} s>1$. The sum is the Dirichlet series and the product is the Euler product. In accordance with the general principle of arithmetic (see, for example, P2]), the definition of $\zeta(s)$ should be extended by adding a factor $\zeta_{\infty}(s)$ at infinity. The new $\zeta$-function will correspond not to the affine scheme $\operatorname{Spec}(\mathbb{Z})$, but to a "complete" scheme $C=$ $\operatorname{Spec}(\mathbb{Z}) \cup \infty$. Precisely as the primes $p$ correspond to $p$-adic completions $\mathbb{Q}_{p}$ of the field $\mathbb{Q}$ (= field of rational functions on $\operatorname{Spec}(\mathbb{Z})), \infty$ corresponds to the embedding of 
$\mathbb{Q}$ into $\mathbb{R}$. The missing factor had been introduced many years before the above ideas emerged. Namely, we put

$$
\zeta_{\infty}(s)=\pi^{-s / 2} \Gamma(s / 2) \quad \text { and } \quad \zeta_{C}(s)=\prod_{x \in C} \zeta_{x}(s) .
$$

Now, let $t \in \mathbb{R}^{*}$, let $f_{t}(x):=\exp \left(-\pi x^{2} t\right) \in \mathcal{S}(\mathbb{R})$, and let

$$
f(t)=\sum_{n \geq 1} \exp \left(-\pi n^{2} t\right)=\frac{1}{2}\left(\sum_{n \in Z} f_{t}(n)-1\right) \text {. }
$$

Then

$$
F\left(f_{t}\right)=t^{-1 / 2} f_{t^{-1}}
$$

For the gamma function, we know that $\Gamma(s)=\int_{0}^{\infty} \exp (-t) t^{s} \frac{d t}{t}$. Then, if we use the Dirichlet series for $\zeta(s)$ and make the change of variables $t \mapsto \pi n^{2} t$, we easily get

$$
\zeta_{C}(s)=\int_{\mathbb{R}_{+}^{*}} t^{s / 2} f(t) \frac{d t}{t} .
$$

Breaking the domain of integration $\mathbb{R}_{+}^{*}$ into two pieces $t<1$ and $t>1$, and applying the simplest Poisson formula to the function $f_{t}(x)$, we arrive at the following expression for the zeta-function 3 :

$$
\zeta_{C}(s)=-\frac{1}{s}+\frac{1}{s-1}+\int_{1}^{\infty} f(t)\left[t^{\frac{s}{2}}+t^{\frac{1-s}{2}}\right] \frac{d t}{t},
$$

where the integral over $t>1$ converges for all $s \in \mathbb{C}$. This gives an analytic continuation of $\zeta_{C}(s)$ and $\zeta(s)$ to the entire $s$-plane $\mathbb{C}$ and shows that the singularities are poles at $s=0$ and $s=1$. The functional equation has the form

$$
\zeta_{C}(1-s)=\zeta_{C}(s) \text {. }
$$

This approach is due to Riemann $[\underline{\mathrm{R}}$ and was extended to zeta- and $L$-functions of the fields of algebraic numbers (= finite extensions of $\mathbb{Q}$ ) by Hecke $[\mathrm{H1}$, $\mathrm{H} 2$, who used a functional equation for the theta-functions (see the Appendix below). In our case, the function $f(t)$ is a simple example of a theta-function. In the next sections we shall study this construction for the parallel case of algebraic curves defined over a finite field. After that, we shall return to number fields.

\section{$\S 2$. The Tate-Iwasawa method for algebraic Curves}

2.1. Preliminaries and notation. Let $C$ be a smooth projective curve over a finite field $k=\mathbb{F}_{q}$. Let $K=k(C)$ be the field of rational functions on $C$. Denote by $\mathbb{A}$ the ring of adeles on $C$, that is,

$$
\mathbb{A}:=\prod_{x \in C}^{\prime} K_{x}
$$

where $x$ runs through all closed points of $C, K_{x}$ is the completion of the field $K$ at the point $x$, and the restricted (adelic) product is taken with respect to the discrete valuation subrings $\widehat{\mathcal{O}}_{x}$ in $K_{x}$. A local parameter $t_{x}$ at a point $x \in C$ determines isomorphisms

$$
K_{x} \cong k(x)\left(\left(t_{x}\right)\right), \quad \hat{\mathcal{O}}_{x} \cong k(x)\left[\left[t_{x}\right]\right],
$$

where $k(x)$ is the residue field of $x$. Furthermore, the field $K_{x}$ is locally compact with respect to the topology defined by the discrete valuation $\nu_{x}$, and $\widehat{\mathcal{O}}_{x}$ is an open compact subring in $K_{x}$. It follows that $\mathbb{A}$ is a topological ring and is locally compact. Recall that

\footnotetext{
${ }^{3}$ We recommend that the reader make all the computations by himself and then compare them with the computations for zeta-functions of curves in the next section. A completely unified exposition of both cases, for number fields and for curves, is given in Weil's book [W2 Chapter VII, §5].
} 
the diagonal embedding $K \hookrightarrow \mathbb{A}$ induces the discrete topology on $K$, while the quotient $\mathbb{A} / K$ is compact. Set $\mathcal{O}:=\prod_{x \in C} \mathcal{O}_{x}$ and, for a divisor $D=\sum_{x \in C} n_{x} \cdot x=\sum_{x \in C} \nu_{x}(D) \cdot x$ on $C$, set

$$
\begin{aligned}
K_{x}(D) & :=\left\{a_{x} \in K_{x} \mid \nu_{x}\left(a_{x}\right)+n_{x} \geq 0\right\}, \\
\mathbb{A}(D) & :=\left\{\left(a_{x}\right) \in \mathbb{A} \mid a_{x} \in K_{x}(D) \text { for all } x \in C\right\} .
\end{aligned}
$$

In particular, $\mathcal{O}=\mathbb{A}(0)$. Note that $\mathbb{A}(D)$ is a compact subset of $\mathbb{A}$.

Let $\mathcal{D}(\mathbb{A})$ be the space of complex-valued Bruhat-Schwartz functions on $\mathbb{A}$, that is, the space of complex-valued locally constant functions with compact support. We view $\mathcal{D}(\mathbb{A})$ as merely a vector space, without any topology. For each divisor $D$ on $C$, the delta-function $\delta_{\mathbb{A}(D)}$ of $\mathbb{A}(D)$ belongs to $\mathcal{D}(\mathbb{A})$. By definition, $\delta_{\mathbb{A}(D)}(a)=1$ if $a \in \mathbb{A}(D)$ and $\delta_{\mathbb{A}(D)}(a)=0$ otherwise.

An important fact is that the space $\mathcal{D}(\mathbb{A})$ is generated by finite linear combinations of the functions $\delta_{\mathbb{A}(D)} 4$

Let $\mathcal{D}^{\prime}(\mathbb{A})$ be the vector space of all complex-valued linear functionals on $\mathcal{D}(\mathbb{A})$, that is, the space of all distributions. An example of a distribution in $\mathcal{D}^{\prime}(\mathbb{A})$ is the delta-function $\delta_{0}, \delta_{0}(f)=f(0)$ for any function $f \in \mathcal{D}(\mathbb{A})$. Another example is the delta-function $\delta_{K}$ of a discrete subset $K \subset \mathbb{A}$ : for any function $f \in \mathcal{D}(\mathbb{A})$, we have

$$
\delta_{K}(f)=\sum_{a \in K} f(a) .
$$

Denote by $\mathbb{A}^{*}$ the group of ideles on $C$, that is, the group of invertible elements in the ring $\mathbb{A}$. We have

$$
\mathbb{A}^{*}=\prod_{x \in C}^{\prime} K_{x}^{*}
$$

where $K_{x}^{*}$ is the multiplicative group of the field $K_{x}$, the adelic product is taken with respect to the subgroups $\widehat{\mathcal{O}}_{x}^{*}$ in $K_{x}^{*}$, and $\widehat{\mathcal{O}}_{x}^{*}$ is the group of invertible elements in the ring $\widehat{\mathcal{O}}_{x}$. As above, $K_{x}^{*}$ is a locally compact topological group, $\widehat{\mathcal{O}}_{x}^{*}$ is an open compact subgroup in $K_{x}^{*}$, and $\mathbb{A}^{*}$ is a locally compact topological group. It can be shown directly that the embedding $\mathbb{A}^{*} \subset \mathbb{A}$ has dense image. Furthermore, there is a well-defined degree homomorphism

$$
\operatorname{deg}: \mathbb{A}^{*} \rightarrow \mathbb{Z}, \quad\left(a_{x}\right) \mapsto \operatorname{deg}\left(a_{x}\right):=\sum_{x \in C} \nu_{x}\left(a_{x}\right) \operatorname{deg}(x),
$$

where $\operatorname{deg}(x)$ is the degree of the residue field $k(x)$ over $k=\mathbb{F}_{q}$ for any closed point $x \in C$. We have $q^{x}:=\# k(x)=q^{\operatorname{deg}(x)}$. Note that the map deg is surjective 5 Let $\left(\mathbb{A}^{*}\right)^{(1)}$ be the kernel of the degree homomorphism. Recall that the diagonal embedding $K^{*} \hookrightarrow \mathbb{A}^{*}$ induces the discrete topology on $K^{*}$, and the restriction of the degree to $K^{*}$ is identically zero. An important result is that the quotient $\left(\mathbb{A}^{*}\right)^{(1)} / K^{*}$ is compact (this corresponds to the fact that degree zero line bundles on $C$ are parametrized by the $\mathbb{F}_{q^{-}}$ points of the Jacobian variety, which is a finite set). Subsequently, we shall deduce this result from the properties of the zeta-function.

\footnotetext{
${ }^{4}$ This fact is well known, see [W2]. It can be deduced, for example, from the presentation of the space $\mathcal{D}(\mathbb{A})$ as a double inductive limit of the spaces $\mathcal{F}\left(\mathbb{A}(D) / \mathbb{A}\left(D^{\prime}\right)\right)$ of all functions on finite sets $\mathbb{A}(D) / \mathbb{A}\left(D^{\prime}\right)$ P1, OP1].

${ }^{5}$ This is not a trivial claim. It means that any curve $C$ contains a divisor of degree 1 (!) but not necessarily a point $x$ of degree 1 , i.e., rational over $\mathbb{F}_{q}$. Indeed, the variety $\operatorname{Pic}^{1}(C)$ parametrizes the classes of divisors of degree 1 . This variety is a torsor for the algebraic group $\mathrm{Pic}^{0}(C)$, and any torsor for a group over a finite field has a rational point; see [S1 Chapter VI, §1, no. 4].
} 
Note that each idele $g \in \mathbb{A}^{*}$ gives rise to distributions $\delta_{g K} \in \mathcal{D}^{\prime}(\mathbb{A})$ and $\delta_{g K^{*}} \in \mathcal{D}^{\prime}(\mathbb{A})$ : for any function $f \in \mathcal{D}(\mathbb{A})$, we have

$$
\delta_{g K}(f)=\sum_{a \in g K} f(a), \quad \delta_{g K^{*}}(f)=\sum_{a \in g K^{*}} f(a)=\delta_{g K}(f)-\delta_{0}(f) .
$$

We shall consider continuous complex-valued characters of the group $\mathbb{A}^{*}$ whose restriction to $K^{*}$ is trivial:

$$
\chi: \mathbb{A}^{*} \longrightarrow \mathbb{C}^{*},\left.\chi\right|_{K^{*}} \equiv 1 .
$$

In particular, the norm homomorphism $|\cdot|:=q^{-\operatorname{deg}(\cdot)}$ is a character of this type. We use the following involution on characters:

$$
\breve{\chi}:=|\cdot| \chi^{-1} .
$$

Since the extension of topological commutative groups

$$
1 \rightarrow\left(\mathbb{A}^{*}\right)^{(1)} / K^{*} \longrightarrow \mathbb{A}^{*} / K^{*} \stackrel{\operatorname{deg}}{\longrightarrow} \mathbb{Z} \rightarrow 0
$$

is split and the group $\left(\mathbb{A}^{*}\right)^{(1)} / K^{*}$ is compact, any character $\chi$ as above can be written as

$$
\chi=\chi_{0}|\cdot|^{s}
$$

for some $s \in \mathbb{C}$, where $\left|\chi_{0}(a)\right|=1$ for any element $a \in \mathbb{A}^{*}$. Therefore,

$$
\left.\operatorname{Hom}\left(\mathbb{A}^{*}\right) / K^{*}, \mathbb{C}^{*}\right)=\mathbb{C}^{*}\left(=\mathbb{C} / \frac{2 \pi i}{\ln q} \mathbb{Z}\right) \times(\text { a discrete group }) .
$$

It follows that the real part $\operatorname{Re}(s)$ of $s$ does not depend on the decomposition above. We denote by $\operatorname{Re}(\chi)$ the real number $\operatorname{Re}(s)$.

2.2. Duality and the Fourier transform. We choose a nonzero rational differential form $\omega$ on $C$ and consider the pairing on $\mathbb{A}$,

$$
\langle\cdot, \cdot\rangle: \mathbb{A} \times \mathbb{A} \rightarrow \mathbf{U}(1),
$$

given by the formula

$$
\left\langle\left(a_{x}\right),\left(b_{x}\right)\right\rangle:=\sum_{x \in C} \Psi\left(\operatorname{Tr}_{k(x) / k} \operatorname{res}_{x}\left(a_{x} b_{x} \omega\right)\right),
$$

where $\mathbf{U}(1)$ can be viewed as the unit circle in $\mathbb{C}$, and $\Psi: k=\mathbb{F}_{q} \rightarrow \mathbb{C}^{*}$ is an injective group homomorphism determined by the choice of a $q$ th root of unity in $\mathbb{C}$. This pairing is continuous and nondegenerate, and the locally compact commutative group $\mathbb{A}$ is its own Pontryagin dual. It is easy to check that the orthogonal of $\mathbb{A}(D)$ with respect to the pairing $\langle\cdot, \cdot\rangle$ is $\mathbb{A}((\omega)-D)$, where $(\omega)$ is the divisor of the differential form $\omega$. A nontrivial fact is that the discrete subgroup $K \subset \mathbb{A}$ is self-orthogonal with respect to the pairing $\langle\cdot, \cdot\rangle$ (this corresponds to the Serre duality on $C$ ), $K^{\perp}=K$. It follows that for any element $g \in \mathbb{A}^{*}$, the annihilator of $g K$ is $g^{-1} K,(g K)^{\perp}=g^{-1} K$.

Also, we choose an additive Haar measure $\mu$ on $\mathbb{A}$. The Fourier transform is defined as usual by the formula

$$
(\mathrm{F} f)(a)=\int_{\mathbb{A}}\langle a, b\rangle f(b) d \mu(b) .
$$

Note that for any function $f \in \mathcal{D}(\mathbb{A})$, the Fourier transform $\mathrm{F}(f)$ is well defined and belongs to $\mathcal{D}(\mathbb{A})$. Hence, the Fourier transform $\mathrm{F}$ is well defined on the dual space $\mathcal{D}^{\prime}(\mathbb{A})$ by the formula

$$
(\mathrm{F} \Delta)(f):=\Delta\left(\mathrm{F}^{-1} f\right)
$$

for all $\Delta \in \mathcal{D}^{\prime}(\mathbb{A})$ and $f \in \mathcal{D}(\mathbb{A})$. The Fourier transform depends on the choice of the differential form $\omega$. Namely, for $\mathrm{F}=\mathrm{F}_{\omega}$ we have

$$
\left(\mathrm{F}_{\omega^{\prime}} f\right)(x)=\left(\mathrm{F}_{\omega} f\right)(a x) \quad \text { if } \quad \omega^{\prime}=a \omega .
$$


Furthermore, the Poisson summation formula and self-orthogonality of $K$ imply that

$$
\begin{aligned}
\mathrm{F} \delta_{K} & =\mu(\mathbb{A} / K)^{-1} \delta_{K}, \\
\mathrm{~F} \delta_{g K} & =|g|^{-1} \mu(\mathbb{A} / K)^{-1} \delta_{g^{-1} K},
\end{aligned}
$$

because $\mu(\mathbb{A} / g K)=|g| \mu(\mathbb{A} / K)$.

It is convenient to work with a self-dual measure $\mu$ on $\mathbb{A}$, which means by definition that for all functions $f, f^{\prime} \in \mathcal{D}(\mathbb{A})$, we have

$$
\mathrm{F}(\mathrm{F} f)(a)=f(-a), \quad \int_{\mathbb{A}} f(a) \overline{f^{\prime}}(a) d \mu(a)=\int_{\mathbb{A}} \mathrm{F} f(a) \overline{\mathrm{F} f^{\prime}}(a) d \mu(a),
$$

where the bar denotes complex conjugation and the Fourier transform is taken with respect to $\mu$. Recall that a self-dual measure $\mu$ on $\mathbb{A}$ exists and is unique. Thus, for a symmetric function $f$ and a symmetric distribution $\Delta 6$, we have $\mathrm{F}(\mathrm{F} f)=f$ and $\mathrm{F}(\mathrm{F} \Delta)=\Delta$. Also, it follows that $\mu(\mathbb{A} / K)=1$, so that $F \delta_{K}=\delta_{K}$ and $F \delta_{g K}=$ $|g|^{-1} \delta_{g^{-1} K}$. Moreover, it can be shown that $\mu(\mathcal{O})=q^{1-g(C)}$, where $g(C)$ is the genus of $C$. Locally, this means that $\mu\left(\mathcal{O}_{x}\right)=q_{x}^{-1 / 2 \nu_{x}(\omega)}$, and for a divisor $D$ on $C$, we have $\mu(\mathbb{A}(D))=q^{1-g(C)+\operatorname{deg}(D)}$. This implies

$$
\begin{aligned}
\mathrm{F} \delta_{K_{x}(D)} & =q_{x}^{-1 / 2 \nu_{x}(\omega)+\nu_{x}(D)} \delta_{K_{x}((\omega)-D),}, \\
\mathrm{F} \delta_{\mathbb{A}(D)} & =q^{1-g(C)+\operatorname{deg}(D)} \delta_{\mathbb{A}((\omega)-D),}
\end{aligned}
$$

where $(\omega)=\sum_{x} \nu_{x}(\omega) \cdot x, D=\sum_{x} \nu_{x}(D) \cdot x, \operatorname{deg} D=\sum_{x} \nu_{x}(D) \cdot \operatorname{deg}(x)$.

2.3. The Weil problem. A simple but very important observation is that the topological group $\mathbb{A}^{*}$ acts on the space $\mathbb{A}$ via multiplication. This determines representations of $\mathbb{A}^{*}$ on the complex vector spaces $\mathcal{D}(\mathbb{A})$ and $\mathcal{D}^{\prime}(\mathbb{A})$. Denote by an upper index the action of an element $g \in \mathbb{A}^{*}$ on a function or a distribution. For all elements $g \in \mathbb{A}^{*}, a \in \mathbb{A}$, $f \in \mathcal{D}(\mathbb{A})$, and $\Delta \in \mathcal{D}^{\prime}(\mathbb{A})$, we have

$$
\begin{aligned}
f^{g}(a) & =f\left(g^{-1} a\right), \quad \Delta^{g}(f)=\Delta\left(f^{g^{-1}}\right), \\
(-1)^{*} f(a) & =f(-a), \quad(-1)^{*} \Delta(f)=\Delta\left((-1)^{*} f\right) .
\end{aligned}
$$

In particular, for all $g, h \in \mathbb{A}^{*}$ we have

$$
\delta_{0}^{g}=\delta_{0}, \quad \delta_{h K}^{g}=\delta_{g^{-1} h K}, \quad \delta_{h K^{*}}^{g}=\delta_{g^{-1} h K^{*}} .
$$

Also, it is easy to show that for all $g \in \mathbb{A}^{*}, f \in \mathcal{D}(\mathbb{A})$, and $\Delta \in \mathcal{D}^{\prime}(\mathbb{A})$, we have

$$
\mathrm{F}\left(f^{g}\right)=|g|(\mathrm{F} f)^{g^{-1}}, \quad \mathrm{~F}\left(\Delta^{g}\right)=|g|(\mathrm{F} \Delta)^{g^{-1}} .
$$

Since the group $\mathbb{A}^{*}$ is commutative, the representation in $\mathcal{D}^{\prime}(\mathbb{A})$ decomposes into one-dimensional representations. The so-called Weil problem [W1] is to find an eigendistribution $\Delta_{\chi} \in \mathcal{D}^{\prime}(\mathbb{A})$ for each character $\chi$ as above, that is, to find a distribution $\Delta_{\chi}$ such that

$$
\Delta_{\chi}^{g}=\chi(g) \cdot \Delta_{\chi}
$$

for any element $g \in \mathbb{A}^{*}$. The functional $\Delta_{\chi}$ is unique up to a scalar; see [W1].

This is obvious for its restriction to the subspace $\mathcal{D}(\mathbb{A})^{\mathcal{O}^{*}}$ of functions invariant under multiplication by the elements of $\mathcal{O}^{*}$. Indeed, the space $\mathcal{D}(\mathbb{A})^{\mathcal{O}^{*}}$ is generated by linear combinations of functions $\delta_{\mathbb{A}(D)}=\delta_{\mathcal{O}}^{g}$, where $g \in \mathbb{A}^{*}$ is such that $\mathbb{A}(D)=g^{-1} \mathbb{A}(0)=$ $g^{-1} \mathcal{O}$. Thus, the values of the distribution $\delta_{\chi}$ will be uniquely determined by its value on

\footnotetext{
${ }^{6}$ Here, the word "symmetric" means that $(-1)^{*} f=f$ and $(-1)^{*} \Delta=\Delta$.

${ }^{7}$ At the same time, the problem of describing homogeneous generalized functions was posed and solved in GGPSh Chapter 2].
} 
the function $\delta_{\mathcal{O}}$. Also, it follows that $\Delta_{\chi}$ is a symmetric distribution $\left((-1)^{*} \Delta_{\chi}=\Delta_{\chi}\right)$, provided it exists.

When $\operatorname{Re}(\chi)>1$, the eigendistribution $\Delta_{\chi}$ can be expressed explicitly (up to a scalar) as follows:

$$
\Delta_{\chi}(f)=\int_{\mathbb{A}^{*}} \chi(g) f(g) d \mu^{*}(g),
$$

where $\mu^{*}$ is a multiplicative Haar measure on the locally compact group $\mathbb{A}^{*}$. It is useful to suppose that $\mu^{*}$ is normalized by the condition $\mu^{*}\left(\mathcal{O}^{*}\right)=1$. Then $\mu^{*}$ is the product of local Haar measures $\mu_{x}^{*}$ on locally compact groups $K_{x}^{*}$, normalized by the conditions $\mu_{x}^{*}\left(\mathcal{O}_{x}^{*}\right)=1$.

We can consider a version of the Weil problem for the group $\mathbb{A}^{*}$ acting on itself by multiplication. The solution $\Delta_{\chi}^{*}$ will belong to the space $\mathcal{D}^{\prime}\left(\mathbb{A}^{*}\right)$ and will be given by the integral (13) for a function $f$ in the space $\mathcal{D}\left(\mathbb{A}^{*}\right)$ rather than $\mathcal{D}(\mathbb{A})$. In that case, the integral will converge for all characters $\chi$. Thus, we get a new well-defined distribution $\Delta_{\chi}^{*}$.

The original Weil problem can also be reformulated in the following way. Let $i$ : $\mathbb{A}^{*} \times \mathbb{A} \rightarrow \mathbb{A}$ be the multiplication map. It induces the direct image for function spaces

$$
i_{*}: \mathcal{D}\left(\mathbb{A}^{*}\right) \otimes \mathcal{D}(\mathbb{A}) \rightarrow \mathcal{D}(\mathbb{A}), \quad i_{*}(f \otimes g)=f * g=\int_{\mathbb{A}^{*}} f\left(x^{-1}\right) g(x y) \frac{d \mu(x)}{|x|} .
$$

This map is a an extension of the convolution map on the group $\mathbb{A}^{*}$, and the original Weil problem is to find a distribution $\Delta_{\chi} \in \mathcal{D}^{\prime}(\mathbb{A})$ such that

$$
\left.\Delta_{\chi}(f * g)\right)=\Delta_{\chi}^{*}(f) \cdot \Delta_{\chi}(g), \quad \text { in other words } i^{*} \Delta_{\chi}=\Delta_{\chi}^{*} \otimes \Delta_{\chi} .
$$

Formula (13) also clarifies the relationship between the Weil problem and the zetafunction of $C$. Namely, if $\operatorname{Re}(s)>1, s \in \mathbb{C}$, then

$$
\Delta_{|\cdot|^{s}}\left(\delta_{\mathcal{O}}\right)=\zeta_{C}(s)
$$

provided that $\mu^{*}$ is normalized as above. Indeed, by (13), the left-hand side equals

$$
\prod_{x \in C} \int_{K_{x}^{*}}\left|g_{x}\right|_{x}^{s} \delta_{\mathcal{O}_{x}}\left(g_{x}\right) d \mu_{x}^{*}\left(g_{x}\right)=\prod_{x \in C}\left(\sum_{n \geq 0} q^{-n s \operatorname{deg}(x)}\right)=\prod_{x \in C}\left(1-q^{-s \operatorname{deg}(x)}\right)^{-1},
$$

where $|\cdot|_{x}:=q^{-\operatorname{deg}(x) \nu_{x}(\cdot)}$, and we have used the fact that $\mathcal{O}_{x}=\coprod_{n \geq 0} t_{x}^{n} \mathcal{O}_{x}^{*}$.

A similar calculation shows that

$$
\Delta_{|\cdot|^{s}}\left(\delta_{\mathbb{A}(D)}\right)=q^{\operatorname{deg}(D) s} \zeta_{C}(s) .
$$

We expand the integral (13) into an integral over the quotient $\mathbb{A}^{*} / K^{*}$ of the sums over fibers of the projection $\mathbb{A}^{*} \rightarrow \mathbb{A}^{*} / K^{*}$ :

$$
\Delta_{\chi}(f)=\int_{\mathbb{A}^{*} / K^{*}} \chi(g) \delta_{g K^{*}}(f) d \mu^{*}(g),
$$

where $f \in \mathcal{D}(\mathbb{A}), g$ runs over all cosets in $\mathbb{A}^{*} / K^{*}$, and $\mu^{*}$ denotes also the induced measure on the quotient $\mathbb{A}^{*} / K^{*}$ (recall that the restriction of $\chi$ to $K^{*}$ is trivial). In what follows we shall consider distribution-valued integrals and, thus, omit $f$ in the integral (16).

The main difficulty with the Weil problem is that formula (16) is not well defined when $\operatorname{Re}(\chi) \leq 1$, because the integral does not converge. However, the integral

$$
\int_{\left(\mathbb{A}^{*}\right) \geq 1 / K^{*}} \chi(g) \delta_{g K^{*}} d \mu^{*}(g)
$$

over a "half" of $\mathbb{A}^{*} / K^{*}$ is well defined for any character $\chi$, where $\left(\mathbb{A}^{*}\right)^{\geq 1}$ is the set of ideles $g \in \mathbb{A}^{*}$ with $|g| \geq 1$. Indeed, as above, the integral (17) converges when $\operatorname{Re}(\chi)>1$. Moreover, if $|g| \geq 1$, then $|\chi(g)| \leq|\chi(g)||g|^{N}$ for any $N>0$, and we have 
$\operatorname{Re}\left(\chi|\cdot|^{N}\right)>1$ for sufficiently large $N$. Hence, one can bound the integral (17) from above for any character $\chi$ by the convergent integral associated with the character $\chi|\cdot|{ }^{s}$ for a suitable $s$.

2.4. Regularization. To overcome the convergence problem in the integral (16), we introduce a regularization of this integral.

Namely, let $\varphi$ be a function on $\mathbb{A}^{*} / K^{*}$ induced by a complex-valued function with finite support on $\mathbb{Z}$ via the degree homomorphism

$$
\operatorname{deg}: \mathbb{A}^{*} / K^{*} \rightarrow \mathbb{Z}
$$

Thus, $\varphi$ has compact support and is locally constant on $\mathbb{A}^{*} / K^{*}$, in other words, $\varphi \in$ $\mathcal{D}\left(\mathbb{A}^{*} / K^{*}\right)$. Define

$$
\Delta_{\chi}^{\varphi}:=\int_{\mathbb{A}^{*} / K^{*}} \varphi(g) \chi(g) \delta_{g K^{*}} d \mu^{*}(g) .
$$

It is easily seen that $\Delta_{\chi}^{\varphi}$ is well defined for any character $\chi$ and that for any function $f \in \mathcal{D}(\mathbb{A})$, the value of $\Delta_{\chi}^{\varphi}(f)$ is a Laurent polynomial in $q^{-s}$.

Consider the limit distribution $\lim _{\varphi \rightarrow 1} \Delta_{\chi}^{\varphi}$, where $\varphi$ tends to the constant function with value 1 , which means that $\varphi(g)=1$ for all $g$ with $|\operatorname{deg}(g)| \leq N$, for increasing values of $N$. One can prove directly that if $\operatorname{Re}(\chi)>1$, then this limit exists and is

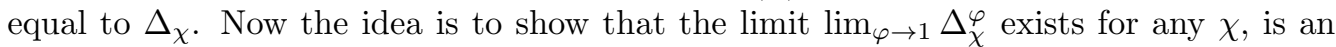
eigendistribution with respect to $\mathbb{A}^{*}$, and also satisfies a certain functional equation.

It is convenient to consider the decomposition $\varphi=\varphi_{-}+\varphi_{+} 8$ where for any $g \in \mathbb{A}^{*} / K^{*}$ we have $\varphi_{-}(g)=\varphi(g)$ if $\operatorname{deg}(g)<0, \varphi_{-}(g)=\varphi(g) / 2$ if $\operatorname{deg}(g)=0$, and $\varphi_{-}(g)=0$ if $\operatorname{deg}(g)>0$. This defines the second function $\varphi_{+}$uniquely.

We have

where

$$
\Delta_{\chi}^{\varphi}=\Delta_{\chi}^{\varphi_{-}}+\Delta_{\chi}^{\varphi_{+}}=\Delta_{\chi}^{\varphi_{-}}+\int_{\mathbb{A}^{*} / K^{*}} \varphi_{+}(g) \chi(g) \delta_{g K} d \mu^{*}(g)-\delta_{0} \cdot c\left(\varphi_{+}, \chi\right),
$$

$$
c\left(\varphi_{+}, \chi\right):=\int_{\mathbb{A}^{*} / K^{*}} \varphi_{+}(g) \chi(g) d \mu^{*}(g) \in \mathbb{C} .
$$

Suppose in addition that $\varphi$ is symmetric, that is, $\varphi(g)=\varphi\left(g^{-1}\right)$. It follows that $\varphi_{ \pm}(g)=\varphi_{\mp}\left(g^{-1}\right)$. Using the Poisson summation formula (8) and the decomposition $g^{-1} K=g^{-1} K^{*} \cup\{0\}$, we obtain

$$
\begin{aligned}
\int_{\mathbb{A}^{*} / K^{*}} & \varphi_{+}(g) \chi(g) \delta_{g K} d \mu^{*}(g) \\
= & \int_{\mathbb{A}^{*} / K^{*}} \varphi_{+}(g) \chi(g)|g|^{-1} \mathrm{~F} \delta_{g^{-1} K} d \mu^{*}(g) \\
= & \int_{\mathbb{A}^{*} / K^{*}} \varphi_{+}(g) \chi(g)|g|^{-1} \mathrm{~F} \delta_{g^{-1} K^{*}} d \mu^{*}(g)+\left(\mathrm{F} \delta_{0}\right) \cdot c\left(\varphi_{+}, \breve{\chi}^{-1}\right) \\
= & \int_{\mathbb{A}^{*} / K^{*}} \varphi_{-}(g) \chi(g)^{-1}|g| \mathrm{F} \delta_{g K^{*}} d \mu^{*}(g)+\left(\mathrm{F} \delta_{0}\right) \cdot c\left(\varphi_{+}, \breve{\chi}^{-1}\right) \\
= & \mathrm{F} \Delta_{\chi_{-}}^{\varphi_{-}}+\left(\mathrm{F} \delta_{0}\right) \cdot c\left(\varphi_{+}, \breve{\chi}^{-1}\right) .
\end{aligned}
$$

In the second line, we replaced $g$ by $g^{-1}$ and made use of the fact that $d \mu^{*}\left(g^{-1}\right)=d \mu^{*}(g)$. Note that the Fourier transform is taken with respect to the self-duality of $\mathbb{A}$ provided

\footnotetext{
${ }^{8}$ This corresponds to Riemann's decomposition of the integration domain of the integral defining the zeta-function $\zeta_{C}(s)$ as the union of two pieces, $t<1$ and $t>1$; see above. More precisely, this corresponds to the approximation of the integration domain by the intervals $N^{-1}<t<1$ and $1<t<N$, and letting $N \rightarrow \infty$ at the end of computations.
} 
by a nonzero rational differential form $\omega$ and the self-dual additive measure $\mu$ on $\mathbb{A}$ (so that $\mu(\mathbb{A} / K)=1)$. Taking the sum, we get

$$
\Delta_{\chi}^{\varphi}=\Delta_{\chi}^{\varphi_{-}}+\mathrm{F} \Delta_{\widetilde{\chi}}^{\varphi_{-}}+\left(\mathrm{F} \delta_{0}\right) \cdot c\left(\varphi_{+}, \breve{\chi}^{-1}\right)-\delta_{0} \cdot c\left(\varphi_{+}, \chi\right) .
$$

Since the integral (17) converges for any character $\chi$, the limits $\Delta_{\chi}^{-}:=\lim _{\varphi \rightarrow 1} \Delta_{\chi}^{\varphi_{-}}$and $\mathrm{F} \Delta_{\bar{\chi}}^{-}=\lim _{\varphi \rightarrow 1} \mathrm{~F} \Delta_{\widetilde{\chi}}^{\varphi_{-}}$are well defined for any character $\chi$. In addition, for a given function $f \in \mathcal{D}(\mathbb{A})$, the sequence $\Delta_{\chi}^{\varphi_{-}}(f)$ will stabilize as $\varphi \rightarrow 1$.

Furthermore, recall that the integral of a character on a compact commutative group equals zero if this character is nontrivial and equals the volume of the group if this character is trivial. This fact, together with a direct calculation, implies that $c\left(\varphi_{+}, \chi\right)=0$ if $\chi$ is not equal to $|\cdot|^{s}$ for some $s \in \mathbb{C}$, and that

$$
c\left(\varphi_{+},|\cdot|^{s}\right)=\mu^{*}\left(\left(\mathbb{A}^{*}\right)^{(1)} / K^{*}\right) \cdot\left(\sum_{n \in \mathbb{Z}} \varphi_{+}\left(g_{n}\right) q^{-n s}\right),
$$

where $g_{n} \in \mathbb{A}^{*} / K^{*}$ with $\operatorname{deg}\left(g_{n}\right)=n$. Therefore, the limit $c(\chi):=\lim _{\varphi \rightarrow 1} c\left(\varphi_{+}, \chi\right)$ equals zero unless $\chi=|\cdot|^{s}$ for some $s \in \mathbb{C}$, and

$$
c\left(|\cdot|^{s}\right)=\mu^{*}\left(\left(\mathbb{A}^{*}\right)^{(1)} / K^{*}\right) \cdot \frac{1+q^{-s}}{1-q^{-s}} .
$$

It particular, $c(\chi)=-c\left(\chi^{-1}\right)$.

By (18), we find that the limit $\Delta_{\chi}:=\lim _{\varphi \rightarrow 1} \Delta_{\chi}^{\varphi}$ is well defined and equals

$$
\Delta_{\chi}^{-}+\mathrm{F} \Delta_{\bar{\chi}}^{-}+\left(\mathrm{F} \delta_{0}\right) \cdot c\left(\breve{\chi}^{-1}\right)-\delta_{0} \cdot c(\chi) .
$$

Making use of formulas (11) and (12), we see that $\Delta_{\chi}$ is indeed an eigendistribution with the character $\chi$. Also, we obtain the functional equation for distributions

$$
\Delta_{\chi}=\mathrm{F} \Delta_{\bar{\chi}} 9
$$

Note that up to a scalar factor this equation also follows from formulas (11) and (12), and the fact that $\Delta_{\chi}$ is an eigendistribution. Thus, we have shown that the Weil problem has a solution for each character $\chi$.

2.5. Applications. This has the following application to the zeta-function of $C$. First, since $\Delta_{|\cdot|^{s}}\left(\delta_{\mathcal{O}}\right)=\zeta_{C}(s)$ for $\operatorname{Re}(s)>1$, we deduce that $\zeta_{C}(s)$ has a meromorphic continuation on the entire $s$-plane. Moreover, formulas (10) and (15) imply that the functional equation

$$
\zeta_{C}(s)=q^{g(C)-1} q^{(2-2 g(C))(s)} \zeta_{C}(1-s)
$$

is satisfied, because $\operatorname{deg}((\omega))=2 g(C)-2$. Finally, it is easily seen that

$$
\mu^{*}\left(\left(\mathbb{A}^{*}\right)^{(1)} / K^{*}\right)=\frac{\left|\operatorname{Pic}^{0}(C)\left(\mathbb{F}_{q}\right)\right|}{q-1},
$$

provided that $\mu^{*}\left(\mathcal{O}^{*}\right)=1$, where $\operatorname{Pic}^{0}(C)$ is the group of isomorphism classes of degree zero line bundles on $C$, and $\operatorname{Pic}^{0}(C)\left(\mathbb{F}_{q}\right)$ is its group of rational points. Indeed, we have the exact sequence

$$
1 \rightarrow \mathbb{F}_{q}^{*} \rightarrow \mathcal{O}^{*} \rightarrow\left(\mathbb{A}^{*}\right)^{(1)} / K^{*} \rightarrow \operatorname{Pic}^{0}(C)\left(\mathbb{F}_{q}\right) \rightarrow 1,
$$

\footnotetext{
${ }^{9}$ This equation shows that the right-hand side does not depend on the choice of the differential form $\omega$ involved in the definition of the Fourier transform. This fact follows easily from the behavior of the distribution under translation and from the triviality of the character $\chi$ on the subgroup $K^{*}$.
} 
the group $\mathcal{O}^{*}$ is compact (a trivial assertion), and the group $\operatorname{Pic}^{0}(C)\left(\mathbb{F}_{q}\right)$ is finite (a much less trivial one). Therefore, the only singularities of $\zeta_{C}(s)$ are two first order poles at $s=0$ and $s=1$, with the residues

$$
\operatorname{res}_{s=0} \zeta_{C}(s)=-\frac{\left|\operatorname{Pic}^{0}(C)\left(\mathbb{F}_{q}\right)\right|}{q-1} \ln ^{-1} q
$$

and

$$
\underset{s=1}{\operatorname{res}} \zeta_{C}(s)=q^{1-g(C)} \frac{\left|\operatorname{Pic}^{0}(C)\right|\left(\mathbb{F}_{q}\right)}{q-1} \ln ^{-1} q .
$$

Also, we can easily deduce the following presentation for $\zeta_{C}(s)$ :

$$
\zeta_{C}(s)=\frac{P\left(q^{-s}\right)}{\left(1-q^{-s}\right)\left(1-q^{1-s}\right)},
$$

where $P\left(q^{-s}\right)$ is a polynomial in the variable $t=q^{-s}$ of degree $2 g(C)$ (see the details in [W2, Chapter VII, $\S 6$, Theorem 4]). This polynomial satisfies the functional equation $P\left(q^{-1} t^{-1}\right)=q^{-g(C)} t^{-2 g(C)} P(t)$. Also, we can remove the singularities, obtaining another presentation for $\zeta_{C}(s)$ :

$$
\begin{aligned}
-\frac{\left|\operatorname{Pic}^{0}(C)\left(\mathbb{F}_{q}\right)\right|}{q-1} \frac{1}{1-q^{-s}} & +q^{1-g(C)} \frac{\left|\operatorname{Pic}^{0}(C)\right|\left(\mathbb{F}_{q}\right)}{q-1} \frac{1}{1-q^{1-s}} \\
& +\int_{\mathbb{A}_{-}^{*}}\left[|g|^{s} f(g)+|g|^{1-s} \mathrm{~F}^{-1} f(g)\right] d \mu^{*}(g),
\end{aligned}
$$

where the integral over the "domain" $\mathbb{A}_{-}^{*}$ means the sum of the integrals over $\left\{g \in \mathbb{A}^{*}\right.$ : $|g|>1\}$ and $1 / 2$ of the integral over $\left\{g \in \mathbb{A}^{*}:|g|=1\right\}$. The integrands will be $f(g)=\delta_{\mathcal{O}}$ and $\mathrm{F}^{-1} f(g)=\mathrm{F} f(g)=q^{1-g(C)} \delta_{\mathbb{A}((\omega))}$.

To see the analogy with the case of Riemann's zeta function (44), we note that $\ln q /(1-$ $\left.q^{-s}\right) \sim 1 / s$ and $\ln q /\left(1-q^{1-s}\right) \sim 1 /(s-1)$. The above presentation will be referred to as the principal parts decomposition of the meromorphic function $\zeta_{C}(s)$.

In our discussion, we implicitly supposed that the group $\left(\mathbb{A}^{*}\right)^{(1)} / K^{*}$ is compact. This fact is nontrivial. It includes both the finiteness of the class number (for number fields and $\operatorname{Pic}^{0}(C)\left(\mathbb{F}_{q}\right)$ in our case) and the Dirichlet theorem on units (see [A, W2]).

It is remarkable and very important that these finiteness results are consequences of the analytical computations we have already carried out. Namely, we can apply the two parts of (18) to a given function $f$ and set $\chi=|\cdot|^{s}$; then (18) can be rewritten as

(polynomial in $\left.q^{-s}\right)=\left(\right.$ polynomial in $\left.q^{-s}\right)-\delta_{(0)}(f) c\left(\varphi_{+},|\cdot|^{s}\right)+\delta_{(0)}(F f) c\left(\varphi_{+},|\cdot|^{1-s}\right)$.

Choosing the function $f$ in an appropriate way, we get

(polynomial in $\left.q^{-s}\right)=\left(\right.$ polynomial in $\left.q^{-s}\right)+\left(\right.$ nonzero polynomial in $\left.q^{-s}\right) \int_{\left(\mathbb{A}^{*}\right)^{(1)} / K^{*}} 1$.

Thus, the volume of $\left(\mathbb{A}^{*}\right)^{(1)} / K^{*}$ is finite and the group itself is compact (see the exact sequence (19) above for this group). This observation was made by Iwasawa [2].

Scholium 1. An important remark is that the entire analytic structure ("singularities" and "principal parts") and the symmetry properties of zeta- and $L$-functions can be visualized without analytic continuation. They are present in their entirety even for the regularized zeta-function before going to the final limit 10

\footnotetext{
${ }^{10}$ That is, $\varphi \rightarrow 1$.
} 


\section{§3. DiscRete VERSIONS AND HOLOMORPHIC TORI}

3.1. Analysis on discrete groups. Let $C / \mathbb{F}_{q}$ be again an algebraic curve defined over a finite field $\mathbb{F}_{q}$. On $C$ we have the following (discrete) groups of divisors:

$$
\Gamma=\operatorname{Div}(C) \supset \Gamma_{0}=\operatorname{Div}^{0}(C) \supset \Gamma_{l}=\operatorname{Div}_{l}(C),
$$

where $\operatorname{Div}^{0}(C)$ is the group of divisors of degree 0 and $\operatorname{Div}_{l}(C)$ is the group of divisors linearly equivalent to 0 . As is well known, $\Gamma_{0} / \Gamma_{l}$ is a finite group $\operatorname{Pic}^{0}(C)\left(\mathbb{F}_{q}\right)$.

Before the Tate-Iwasawa approach was developed, another method had been known for the case of algebraic curves. It was based on a direct application of the Riemann-Roch theorem (see, for example, $[\mathrm{D}]$ ). Denote by $\Gamma_{+}=\{D \in \Gamma: D \geq 0\}$ the subset of effective divisors on $C$. The zeta-function can be written as the Dirichlet series converging for $\operatorname{Re}(s)>1$,

$$
\zeta_{C}(s)=\sum_{D \in \Gamma_{+}} \operatorname{Nm}(D)^{-s}, \text { where } \operatorname{Nm}(D)=q^{\operatorname{deg} D} .
$$

Assuming for simplicity that there is a point $P \in C$ such that $k(P)=\mathbb{F}_{q}$ and choosing a finite set of representatives $R$ of cosets of $\Gamma_{l}$ in $\Gamma_{0}$, we have the following decompositions:

$$
\Gamma=\coprod_{R, n \in \mathbb{Z}}(R+n P) \Gamma_{l}, \quad \Gamma_{+}=\coprod_{R, n \in \mathbb{Z}}(R+n P) \Gamma_{l} \cap \Gamma_{+} .
$$

Therefore, the Dirichlet series can be transformed a: 11

$$
\begin{aligned}
\zeta_{C}(s) & =\sum_{R, n \in \mathbb{Z}} \#\{D \in \Gamma: D \sim R+n P, D \geq 0\} q^{-n s}=\sum_{R, n \geq 0} \frac{q^{l(R+n P)}-1}{q-1} q^{-n s} \\
& =-\frac{1}{q-1} \sum_{R, n \geq 0} q^{-n s}+\frac{1}{q-1} \sum_{R, n \geq 0} q^{l(R+n P)} q^{-n s}=? .
\end{aligned}
$$

Now, we come to the main point, which is to use duality and the Riemann-Roch theorem together: $l(R+n P)=l(\mathrm{~K}-R-n P)+n+1-g(C)$. Here, $\mathrm{K}=(\omega), \omega \in \Omega_{K}^{1}$ is the canonical class, and we choose $\omega \in \Omega_{K}^{1}$ such that $\mathrm{K}=(2 g-2) P+R_{0}$ with $g=g(C), R_{0} \in\{R\}$. Note that $\left\{R_{0}-R\right\}=\{S\}$ where $S$ is another finite set of representatives of the cosets of $\Gamma_{l}$ in $\Gamma_{0}$. We continue the transformation of the zeta-series:

$$
\begin{aligned}
= & -\frac{1}{q-1} \sum_{R, n \geq 0} q^{-n s}+\frac{q^{1-g}}{q-1} \sum_{R, n \geq 0} q^{l\left(R_{0}-R+(2 g-2-n) P\right)} q^{-n(s-1)} \\
= & -\frac{1}{q-1} \sum_{R, n \geq 0} q^{-n s}+\frac{q^{1-g}}{q-1} \sum_{S, n \geq 0} q^{-n(s-1)} \\
& +\frac{q^{1-g}}{q-1} \sum_{S, 2 g-2 \geq n \geq 0}\left(q^{l(R+(2 g-2-n) P)}-1\right) q^{-n(s-1)}
\end{aligned}
$$

At the end, we get a sum of three terms. The first has poles at the points of the arithmetical progressions $2 \pi i m / \ln q, m \in \mathbb{Z}$, the second has poles at the points of the arithmetical progressions $1+2 \pi i m / \ln q, m \in \mathbb{Z}$, and the last term is a polynomial in $q^{-s}$. Thus, we have made an analytic continuation of $\zeta_{C}(s)$ to the entire $s$-plane. It is also not difficult to obtain the functional equation; see [D, §23]. It is worthwhile to compare this computations with those made in the previous section. Elaborating upon this approach

\footnotetext{
${ }^{11}$ Here we use two standard notations: $\sim$ for linear equivalence of divisors and $l(D)$ for the dimension of the space $\mathrm{H}^{0}\left(C, \mathcal{O}_{C}(D)\right)$.
} 
in the spirit of the Tate-Iwasawa method, one can also incorporate a function $f=f(D)$ into the Dirichlet series and study more general sums

$$
\zeta_{C}(s, f)=\sum_{D \in \Gamma} f(D) \operatorname{Nm}(D)^{-s} .
$$

Leaving this to the reader, now we introduce and carefully study the necessary tools: function spaces, the Fourier transform, and the Poisson formula.

We filter the group $\Gamma$ by the subgroups $\Gamma_{S}:=\operatorname{Div}_{S}(C)=\{$ divisors with support in $S\}$ :

$$
\Gamma=\underset{S}{\lim } \Gamma_{S}=\underset{S}{\lim } \bigoplus_{x \in S} \Gamma_{(x)}=\bigoplus_{x \in C} \Gamma_{(x)},
$$

where the $S$ are finite subsets of $C$ and $\Gamma_{(x)}=\mathbb{Z}$ is the valuation group at the point $x$.

On $\mathbb{Z}$ there are two important spaces: the space $\mathcal{D}(\mathbb{Z})$ of finitely supported functions and the space $\mathcal{D}_{+}(\mathbb{Z})$ of functions $f$ such that $f(n)=0$ for $n \ll 0$ and $f(n)=$ const for $n \gg 0$.

The space $\mathcal{D}_{+}(\mathbb{Z})$ is the linear span of the functions

$$
\delta_{(\geq m)}(n)= \begin{cases}1 & \text { if } n \geq m \\ 0 & \text { if } n<m .\end{cases}
$$

This function space can be obtained directly from the Bruhat spaces introduced above. The group $\mathbb{Z}=\Gamma_{(x)}$ appears as $K_{x}^{*} / \widehat{\mathcal{O}}_{x}^{*}$, where $K_{x}$ is the local field at the point $x \in C$. Then $K_{x}=\coprod_{n \in \mathbb{Z}} t_{x}^{n} \widehat{\mathcal{O}}_{x}^{*} \cup\{0\}$. Thus, we have the isomorphism

$$
\mathcal{D}\left(K_{x}\right)^{\hat{\mathcal{O}}_{x}^{*} \stackrel{\sim}{\rightarrow}} \mathcal{D}_{+}(\mathbb{Z}), \quad\left\{f \mapsto f\left(t_{x}^{n}\right)\right\},
$$

from the subspace of functions invariant under multiplication by $\widehat{\mathcal{O}}_{x}^{*}$ onto the space of functions on the group $\mathbb{Z}$. In the same way, we have an isomorphism

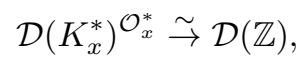

where $\mathcal{D}(\mathbb{Z})$ is the space of functions on $\mathbb{Z}$ with finite support.

As in the case of Bruhat functions, we have the convolution map

$$
*: \mathcal{D}(\mathbb{Z}) \otimes \mathcal{D}_{+}(\mathbb{Z}) \rightarrow \mathcal{D}_{+}(\mathbb{Z})
$$

that sends $f \otimes g$ to the function $f * g$ defined by

$$
f * g(n)=\sum_{m \in \mathbb{Z}} f(n-m) g(m), \quad f \in \mathcal{D}(\mathbb{Z}), g \in \mathcal{D}_{+}(\mathbb{Z}),
$$

and the space $\mathcal{D}_{+}(\mathbb{Z})$ is a free $\mathcal{D}(\mathbb{Z})$-module with the generator $\delta_{(\geq 0)}$.

The Fourier transform $\mathrm{F}=\mathrm{F}_{x}: \mathcal{D}\left(K_{x}\right) \rightarrow \mathcal{D}\left(K_{x}\right)$ commutes with the action of the group $\widehat{\mathcal{O}}_{x}^{*}$; consequently, it induces an analog of the Fourier transform on the group $\mathbb{Z}=\Gamma_{(x)}$ at the point $x$ :

$$
\mathrm{F}_{x}: \mathcal{D}_{+}(\mathbb{Z}) \rightarrow \mathcal{D}_{+}(\mathbb{Z})
$$

Recall that we use a self-dual measure on the local field $K_{x}$ to define the Fourier transform $\mathrm{F}_{x}$. Let $k_{x}:=\nu_{x}(\omega)$ for $\omega \in \Omega_{K}^{1}$. Then, one can easily compute this map on the standard functions:

$$
\mathrm{F}_{x}\left(\delta_{(\geq m)}\right)=q_{x}^{-1 / 2 k_{x}-m} \delta_{\left(\geq-k_{x}-m\right)} .
$$

Here we have used the fact that if $\nu_{x}(D)=n_{x}$, then $\delta_{K_{x}(D)}$ is mapped to $\delta_{\geq-n_{x}}$ under the morphism (20) (see Subsection 2.2). 
Now we globalize these purely local considerations. We need the spaces $\mathcal{D}(\Gamma)$ and $\mathcal{D}_{+}(\Gamma)$ for the group of all divisors. First, we change the notation for the standard functions:

$$
f_{x, D}\left(n_{x}\right)=\left\{\begin{array}{lll}
1 & \text { if } n_{x} \geq \nu_{x}(D) \\
0 & \text { if } n_{x}<\nu_{x}(D)
\end{array}\right.
$$

where $n_{x} \in \Gamma_{(x)}$ and $D$ runs through the divisors on the curve $C$. Sometimes, we shall denote this function by $\delta_{(\geq n)}$ as before, where $n=\nu_{x}(D)$.

Taking the finite products

$$
f_{D}\left(\left(n_{x}\right)_{x \in S}\right)=\bigotimes_{x \in S} f_{x, D}\left(n_{x}\right)
$$

we get functions on $\Gamma_{S}$. We set

$$
\mathcal{D}(\Gamma):=\left\{\text { linear span of the functions } f_{D}=\bigotimes_{x \in \operatorname{Supp} D} \delta_{\left(n_{x}\right)} \bigotimes_{x \notin \operatorname{Supp} D} \delta_{(0)}\right\},
$$

where $\delta_{\left(n_{x}\right)}(n)$ is equal to 1 if $n=n_{x}$ and to 0 if $n \neq n_{x}$. This space consists of all finitely supported functions on $\Gamma$. Also, we have the space

$$
\mathcal{D}_{+}(\Gamma):=\left\{\text { linear span of the functions } f_{D}=\bigotimes_{x \in \operatorname{Supp} D} f_{x, D} \bigotimes_{x \notin \operatorname{Supp} D} \delta_{(\geq 0)}\right\},
$$

which is equal to the space of invariants $\mathcal{D}\left(\mathbb{A}^{*}\right)^{\mathcal{O}^{*}}$ with $\mathcal{O}^{*}=\prod_{x \in C} \mathcal{O}_{x}^{*}$. The functions $f_{D}$ correspond to the functions $\delta_{\mathbb{A}(-D)} \in \mathcal{D}(\mathbb{A})^{\mathcal{O}^{*}}$.

The Poisson formula $\delta_{g K}(f)=|g|^{-1} \delta_{g^{-1} K}(\mathrm{~F} f)$ for the space $\mathcal{D}(\mathbb{A})$ can be formulated in the new situation as follows. 12 For simplicity, first we suppose that $g=1$. Rewrite the Poisson formula as

$$
\delta_{(0)}(f)+\delta_{K^{*}}(f)=\delta_{(0)}(F f)+\delta_{K^{*}}(F f)
$$

and assume that $f$ belongs to $\mathcal{D}(\mathbb{A})^{\mathcal{O}^{*}}$. The group $\Gamma$ contains the subgroup $\Gamma_{l}$, which is the group of divisors of functions $g \in K^{*}$. Thus, $\Gamma_{l}=K^{*} / \mathbb{F}_{q}^{*}$. This means that the delta function $\delta_{K^{*}}(f)$ is equal to $(q-1)$ times the delta-function $\delta_{\Gamma_{l}}(f)$, applied to $f$ viewed as a function in the space $\mathcal{D}_{+}(\Gamma)$. What should we do with the functionals $\delta_{(0)}(f)$ ? The zero subgroup in $\mathbb{A}$ does not correspond to any subset of $\Gamma$, and the functional can be defined in the following way:

$$
\delta_{(0)}(f)=\prod_{x} \delta_{(0)}\left(f_{x}\right) \quad \text { if } \quad f=\bigotimes_{x \in C} f_{x}
$$

with

$$
\delta_{(0)}\left(f_{x}\right)=\lim _{n \rightarrow \infty} f_{x}(n) .
$$

The new Poisson formula for the functions $f \in \mathcal{D}_{+}(\Gamma)$ reads

$$
\delta_{(0)}(f)+(q-1) \delta_{\Gamma_{l}}(f)=\delta_{(0)}(\mathrm{F} f)+(q-1) \delta_{\Gamma_{l}}(\mathrm{~F} f) .
$$

More generally, when $g \neq 1, g \in \mathbb{A}^{*}$, we get

$$
\delta_{(0)}(f)+(q-1) \delta_{\gamma+\Gamma_{l}}(f)=\delta_{(0)}(\mathrm{F} f)+(q-1) \delta_{-\gamma+\Gamma_{l}}(\mathrm{~F} f)
$$

with $\gamma \in \Gamma$ (which is the image of $g$ under the natural map $\mathbb{A}^{*} \rightarrow \Gamma$ ).

${ }^{12}$ Since $-1 \in \mathcal{O}^{*}$, we have $\mathrm{F}=\mathrm{F}^{-1}$ on the spaces $\mathcal{D}(\Gamma)$ and $\mathcal{D}_{+}(\Gamma)$. 
3.2. Extended group of divisors. The presence of the factors $q-1$ can be avoided if we consider an extended adelic group. Let $\mathcal{O}_{x} / \mathfrak{m}_{x}=k(x)$ and set

$$
\widetilde{\Gamma}_{x}:=K_{x}^{*} /\left(1+\mathfrak{m}_{x}\right) .
$$

Then we have a canonical exact sequence:

$$
1 \longrightarrow k(x)^{*} \longrightarrow \widetilde{\Gamma}_{x} \stackrel{\pi}{\longrightarrow} \mathbb{Z} \longrightarrow 0
$$

For a subset $M \subset \mathbb{Z}$, we define $\widetilde{\Gamma}_{x}(M):=\pi^{-1}(M)$. Then we introduce the following space of functions on this new group:

$$
\mathcal{D}_{+}\left(\widetilde{\Gamma}_{x}\right)=\left\{\begin{array}{llll}
f \in \mathcal{F}\left(\widetilde{\Gamma}_{x}\right): & f(\gamma)=\text { const } & \text { on } \widetilde{\Gamma}_{x}(\geq n) & \text { for } n \geq n_{1}, \\
f(\gamma)=0 & \text { on } \widetilde{\Gamma}_{x}(n) & \text { for } n \leq n_{0}
\end{array}\right\} .
$$

Now we can introduce the global group $\widetilde{\Gamma}_{C}=\prod_{x \in C}^{\prime} \widetilde{\Gamma}_{x}$ which fits into the exact sequence

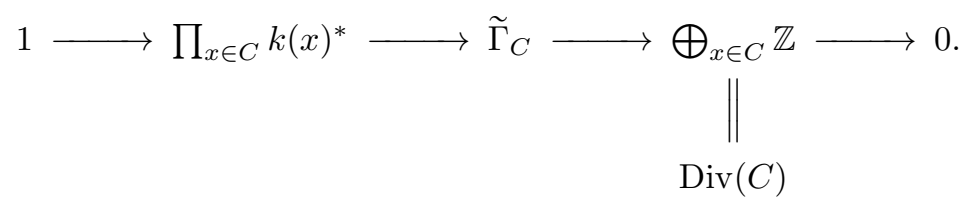

As above, fixing a differential form $\omega \in \Omega_{\mathbb{F}_{q}(C)}^{1}, \omega \neq 0,(\omega)=\sum k_{x} \cdot x$, we define the local Fourier transforms $\mathrm{F}: \mathcal{D}_{+}\left(\widetilde{\Gamma}_{x}\right) \stackrel{\sim}{\rightarrow} \mathcal{D}_{+}\left(\widetilde{\Gamma}_{x}\right)$ and their global counterpart. There is a map $K^{*}=\mathbb{F}_{q}(C)^{*} \rightarrow \widetilde{\Gamma}_{C}$, and if we set $\widetilde{\Gamma}_{l}$ to be the image of $K^{*}$ under this map, we arrive at a new Poisson formula (without the additional factors $q-1$ ):

$$
\delta_{(0)}(f)+\delta_{\gamma+\widetilde{\Gamma}_{l}}(f)=q^{-\operatorname{deg}(\gamma)} \delta_{(0)}(\mathrm{F} f)+q^{-\operatorname{deg}(\gamma)} \delta_{-\gamma+\widetilde{\Gamma}_{l}}(\mathrm{~F} f)
$$

with $\gamma \in \widetilde{\Gamma} / \widetilde{\Gamma}_{l}$. Here, we can define the functionals $\delta_{(0)}(f)$ as we did for the group $\Gamma$.

Indeed, inside the full adelic group we have subgroups $K^{*} \subset \mathbb{A}^{*}$ and $\prod_{x}\left(1+\mathfrak{m}_{x}\right)=$ : $\mathcal{O}_{1}^{*} \subset \mathbb{A}^{*}$. Then $\widetilde{\Gamma}_{C}=\mathbb{A}^{*} / \mathcal{O}_{1}^{*}$ and $K^{*} \cap \mathcal{O}_{1}^{*}=1$. This gives the Poisson formula on $\mathbb{A}_{K}$ for functions $f \in \mathcal{D}(\mathbb{A})^{\mathcal{O}_{1}^{*}}=\mathcal{D}_{+}\left(\widetilde{\Gamma}_{C}\right)$.

There is a measure on $\widetilde{\Gamma}_{C}$ that consists in counting on the discrete group $\Gamma_{C}=$ $\operatorname{Div}(C)$ and integrating over the compact group $\prod_{x} k(x)^{*}$. Then we can make the entire computation of the zeta-function $\zeta_{C}(s)$ using only analysis on the group $\widetilde{\Gamma}_{C} 13$

3.3. Holomorphic tori. The next step in our construction will be to consider the dual groups of the groups such as $\mathbb{Z}=\Gamma_{(x)}$ or $\Gamma_{C}$. The usual Pontryagin definition is not adequate for our arithmetical goals. We wish to view discrete groups as commutative group schemes over $\mathbb{C}$. Inside the category of commutative group schemes we have two subcategories, the category $\mathcal{T}$ of commutative algebraic groups of toroidal type (such that the connected components are tori isomorphic to several copies of $\mathbb{G}_{m}$ ) and the

${ }^{13}$ On the curve $C / \mathbb{F}_{q}$, there is a Heisenberg group $1 \longrightarrow \bigoplus_{x \in C} k(x)^{*} \longrightarrow \widehat{\Gamma}_{C} \longrightarrow \widetilde{\Gamma}_{C} \longrightarrow 1$ or

$$
\left(\begin{array}{ccc}
1 & \bigoplus_{x} \mathbb{Z} & \bigoplus_{x} k(x)^{*} \\
0 & 1 & \prod_{x} k(x)^{*} \\
0 & 0 & 1
\end{array}\right)
$$

if we put $\left(n_{x}\right) \times\left(b_{x}\right) \longmapsto\left(b_{x}^{n_{x}}\right)$ for $\left(n_{x}\right) \in \bigoplus_{x} \mathbb{Z},\left(b_{x}\right) \in \prod_{x} k(x),\left(b_{x}^{n_{x}}\right) \in \bigoplus_{x} k(x)$. What is its arithmetic meaning? Are there some $L$-functions related to this group and, in particular, to characters of its center? 
category $\mathcal{A}$ of finitely generated Abelian groups (viewed as discrete group schemes). We have two functors between them:

$$
\begin{aligned}
& \Theta: \mathcal{T} \rightarrow \mathcal{A}, \quad \Theta(T)=\operatorname{Hom}\left(T, \mathbb{G}_{m}\right), \\
& \Psi: \mathcal{A} \rightarrow \mathcal{T}, \quad \Psi(A)=\operatorname{Hom}\left(A, \mathbb{G}_{m}\right),
\end{aligned}
$$

with the obvious definition of the corresponding maps on arrows. If $G, G^{\prime} \in \mathrm{Ob} \mathcal{A}$ and $f: G \rightarrow G^{\prime}$ is an arrow of $\mathcal{A}$, we denote the dual map by

$$
\check{f}: \Psi\left(G^{\prime}\right) \rightarrow \Psi(G) .
$$

These functors possess all the standard properties of duality maps.

The dual groups of our discrete groups will be complex tori:

$$
\begin{aligned}
& \mathbb{T}_{(x)}:=\operatorname{Hom}\left(\Gamma_{(x)}, \mathbb{C}^{*}\right), \\
& \mathbb{T}_{\Gamma}(\text { or simply } \mathbb{T})=\operatorname{Hom}\left(\Gamma, \mathbb{C}^{*}\right):=\underbrace{\lim }_{S} \operatorname{Hom}\left(\Gamma_{S}, \mathbb{C}^{*}\right), \\
& \mathbb{T}_{\text {Pic }}:=\operatorname{Hom}\left(\Gamma / \Gamma_{l}, \mathbb{C}^{*}\right) \supset \mathbb{T}_{0}:=\operatorname{Hom}\left(\Gamma / \Gamma_{0}, \mathbb{C}^{*}\right)=\mathbb{C}^{*},
\end{aligned}
$$

where inclusion is induced by the degree map: $\Gamma \stackrel{\text { deg }}{\rightarrow} \Gamma / \Gamma_{0}=\mathbb{Z} \mathbb{1 4}$

We have a short exact sequence

$$
1 \rightarrow \mathbb{T}_{0} \rightarrow \mathbb{T}_{\text {Pic }} \rightarrow \operatorname{Hom}\left(\operatorname{Div}^{0}(C) / \operatorname{Div}_{l}(C), \mathbb{C}^{*}\right) \rightarrow 0 ;
$$

therefore, $\mathbb{T}_{\text {Pic }} \cong \mathbb{C}^{*} \times\{$ finite group $\}$. In the sequel, we shall denote the finite group $\operatorname{Div}^{0}(C) / \operatorname{Div}_{l}(C)$ by $\Phi$ and its dual group by $\check{\Phi}$.

In order to regard the torus $\mathbb{T}_{\Gamma}$ as a manifold, we only need to know the ring of regular functions on $\mathbb{T}_{\Gamma}$. For this, we introduce the projective system of tori $\mathbb{T}_{S}:=$ $\operatorname{Hom}\left(\operatorname{Div}_{S}(C), \mathbb{C}^{*}\right)=\prod_{x \in S} \operatorname{Hom}\left(\Gamma_{(x)}, \mathbb{C}^{*}\right)=: \prod_{x \in S} \mathbb{T}_{(x)}$ with a natural projection

$$
\mathbb{T}_{S} \leftarrow \mathbb{T}_{S^{\prime}} \text { if } S \subset S^{\prime},
$$

induced by the embedding $\operatorname{Div}_{S}(C) \subset \operatorname{Div}_{S^{\prime}}(C)$.

Now, consider the divisor $D_{S}$ with normal crossings on $\mathbb{T}_{S}$ that consists of the points in the product $\mathbb{T}_{S}$ for which at least one component is the identity point in some $\mathbb{T}_{(x)}$. Let $\mathbb{C}_{+}\left[\mathbb{T}_{S}\right]$ be the space of rational functions on $\mathbb{T}_{S}$ that are regular outside $D_{S}$ and may have poles of first order on $D_{S}$. In particular, $\mathbb{C}_{+}\left[\mathbb{T}_{(x)}\right]=\mathbb{C}\left[\mathbb{T}_{(x)}\right](1-z)^{-1}$.

Then $\mathbb{T}:=\lim _{S} \mathbb{T}_{S}$, and we set

$$
\begin{aligned}
\mathbb{C}[\mathbb{T}] & :=\underset{S}{\lim } \mathbb{C}\left[\mathbb{T}_{S}\right]=\bigotimes_{x \in C}^{\prime} \mathbb{C}\left[\mathbb{T}_{(x)}\right] \\
& :=\left\{\left(\bigotimes_{x \in C} f_{x}\right): f_{x} \in \mathbb{C}\left[\mathbb{T}_{(x)}\right] \& f_{x}=1 \text { for almost all } x\right\}, \\
\mathbb{C}_{+}[\mathbb{T}]: & =\underset{S}{\lim } \mathbb{C}_{+}\left[\mathbb{T}_{S}\right]=\bigotimes_{x \in C}^{\prime} \mathbb{C}_{+}\left[\mathbb{T}_{(x)}\right] \\
& :=\left\{\left(\bigotimes_{x \in C} f_{x}\right): f_{x} \in \mathbb{C}_{+}\left[\mathbb{T}_{(x)}\right] \& f_{x}=\left(1-z_{x}\right)^{-1} \text { for almost all } x\right\} .
\end{aligned}
$$

Here $z_{x}$ is a coordinate on $\mathbb{T}_{(x)}$, namely, for $\chi \in \mathbb{T}_{(x)}$ we have $z_{x}=\chi(1)$.

It is easily seen that

(1) $\mathbb{C}_{+}[\mathbb{T}]$ is a free module over the ring $\mathbb{C}[\mathbb{T}]$ with generator $\left(f_{x}\right)$, where $f_{x}=$ $\left(1-z_{x}\right)^{-1}$ for all $x$

${ }^{14}$ The complex structure on the set of characters (with values in $\mathbb{C}^{*}$ ) was considered by A. Weil in $\mathrm{W} 2$. 
(2) for all (finite) $S$, we have $\mathbb{C}_{+}\left[\mathbb{T}_{S}\right] \supset \mathbb{C}\left[\mathbb{T}_{S}\right]$ but nevertheless $\mathbb{C}_{+}[\mathbb{T}] \not \supset \mathbb{C}[\mathbb{T}]$.

Assume now that we have an algebraic group $\mathbb{T}$ which is not necessarily connected but whose connected component $\mathbb{T}_{e}$ of the identity $e$ is a torus, and for which $\pi_{0}(\mathbb{T})=\mathbb{T} / \mathbb{T}_{e}$ is a discrete group. Then we know that

$$
\mathbb{C}[\mathbb{T}]=\bigoplus_{h \in \pi_{0}(\mathbb{T})} \mathbb{C}[h \mathbb{T}]
$$

and we set

$$
\mathbb{C}_{+}[\mathbb{T}]=\mathbb{C}_{+}\left[\mathbb{T}_{e}\right] \underset{h \in \pi_{0}(\mathbb{T}), h \neq e}{\bigoplus} \mathbb{C}[h \mathbb{T}] .
$$

Now we would like to describe the relationship between functions on discrete groups and on their duals. Certainly, this must be some kind of Fourier transform, but to distinguish it from the Fourier transform on the original discrete groups we shall call the corresponding transformation the Mellin transform.15 If $f \in \mathcal{F}(\mathbb{Z})$ belongs to a function space on $\mathbb{Z}$, then the Mellin transform $\mathrm{M} f$ will be

$$
\mathrm{M} f(z)=\sum_{n \in \mathbb{Z}} f(n) z^{n}
$$

where $z \in \operatorname{Hom}\left(\mathbb{Z}, \mathbb{C}^{*}\right)$ and $z^{n}$ is $z(n)$. The Mellin transform maps the spaces $\mathcal{D}(\mathbb{Z})$ and $\mathcal{D}_{+}(\mathbb{Z})$ isomorphically onto the spaces $\mathbb{C}\left[\mathbb{T}_{(x)}\right]$ and $\mathbb{C}_{+}\left[\mathbb{T}_{(x)}\right]$, respectively. These isomorphisms allow us to define the Fourier transform, say $\mathrm{F}_{\text {new }}$, on the spaces $\mathbb{C}_{+}\left[\mathbb{T}_{(x)}\right]$ such that $\mathrm{M} \circ \mathrm{F}_{\text {old }}=\mathrm{F}_{\text {new }} \circ \mathrm{M} 16$ The Fourier transforms of the standard functions on $\mathbb{T}_{(x)}$ can be computed in an explicit way. Starting from the identity

$$
\mathrm{F}_{x}\left(f_{x, D}\right)=q_{x}^{-\frac{1}{2} k_{x}+n_{x}} f_{x, \mathrm{~K}-D}
$$

on the group $\Gamma_{x}$, where $D=\sum_{x} n_{x} \cdot x, n_{x}=\nu_{x}(D)$, and $\mathrm{K}=\sum_{x} k_{x} \cdot x$ is the canonical divisor, in the space $\mathbb{C}_{+}\left[\mathbb{T}_{(x)}\right]=\mathbb{C}\left[z_{x}, z_{x}^{-1}\right]\left(1-z_{x}\right)^{-1}$ we get

$$
\mathrm{F}_{x}\left(z_{x}^{m}\left(1-z_{x}\right)^{-1}\right)=q_{x}^{-\frac{1}{2} k_{x}-m} z_{x}^{-\left(k_{x}+m\right)}\left(1-z_{x}\right)^{-1}
$$

for any $m \in \mathbb{Z}$, because

$$
\mathrm{M} f_{x, D}=z_{x}^{\nu_{x}(D)}\left(1-z_{x}\right)^{-1}=z_{x}^{n_{x}}\left(1-z_{x}\right)^{-1} .
$$

Now we can write the Fourier transform in the general form, for an arbitrary function $f$ in $\mathbb{C}_{+}\left[\mathbb{T}_{(x)}\right]$ :

$$
\mathrm{F}_{x}\left(f\left(z_{x}\right)\right)=q_{x}^{-\frac{1}{2} k_{x}} z_{x}^{-k_{x}}\left(1-q_{x}^{-1} z_{x}^{-1}\right)\left(1-z_{x}\right)^{-1} f\left(q_{x}^{-1} z_{x}^{-1}\right) .
$$

We move to the global construction. Applying the Mellin transform to the standard functions, we check that

$$
\mathbb{C}_{+}\left[\mathbb{T}_{S}\right]=\text { linear span of the functions } M f_{D}, \quad D \in \operatorname{Div}_{S}(C) .
$$

We also see that for almost all $x \in C, \mathrm{~F}_{x}\left(\left(1-z_{x}\right)^{-1}\right)=\left(1-z_{x}\right)^{-1}$ in the space $\mathbb{C}\left[\mathbb{T}_{x}\right]$. These facts allow us to combine the local Fourier transforms $\mathrm{F}_{x}$ for all $x$ into a global transformation $\mathrm{F}$ on the torus $\mathbb{T}_{\Gamma}$,

$$
\mathrm{F}: \mathbb{C}_{+}\left[\mathbb{T}_{\Gamma}\right] \stackrel{\sim}{\rightarrow} \mathbb{C}_{+}\left[\mathbb{T}_{\Gamma}\right]
$$

so that $\mathrm{F}\left(f_{x}\right)=\left(\mathrm{F}_{x} f_{x}\right)$ and $\mathrm{F} \circ \mathrm{F}=\mathrm{Id}$.

The exact sequence

$$
0 \rightarrow \Gamma_{0} \rightarrow \Gamma \stackrel{\pi}{\rightarrow} \Gamma / \Gamma_{0} \rightarrow 0
$$

\footnotetext{
${ }^{15}$ Certainly, this usage corresponds to the well-known cases where the classical Mellin map arises.

${ }^{16}$ Here, $F_{\text {old }}$ is the Fourier transform on the space $\mathcal{D}_{+}(\mathbb{Z})$. In the sequel, we shall use one and the same notation $\mathrm{F}$ for the Fourier transforms in all spaces.
} 
determines an embedding of tori:

$$
\check{\pi}: \mathbb{T}_{0} \rightarrow \mathbb{T} .
$$

If $z_{x} \in \mathbb{T}_{x}=\operatorname{Hom}\left(\mathbb{Z}_{(x)}, \mathbb{C}^{*}\right)$ and $\operatorname{Div}(C)=\bigoplus_{x \in C} \mathbb{Z}_{(x)}$, then the embedding $\check{\pi}: \mathbb{T}_{0} \rightarrow \mathbb{T}$ can be written as $z \mapsto z_{x}=z^{\operatorname{deg}(x)}$, where $\# k(x)=q_{x}=q^{\operatorname{deg}(x)}$.

This embedding induces a map of $\mathbb{C}_{+}\left[\mathbb{T}_{\Gamma}\right]$ into some function space

$$
\mathbb{C}_{+}[\mathbb{T}] \stackrel{\check{\pi}^{*}}{\longrightarrow} \mathcal{F}\left(\mathbb{T}_{0}\right)
$$

where we still need to identify the target space! By direct substitution, we get

$$
\begin{aligned}
\check{\pi}^{*}\left(\mathcal{L} f_{D}\right) & =\prod_{x \in C}\left(z^{\operatorname{deg}(x)}\right)^{\nu_{x}(D)}\left(1-z^{\operatorname{deg}(x)}\right)^{-1} \\
& =z^{\operatorname{deg} D} \prod_{x}\left(1-z^{\operatorname{deg}(x)}\right)^{-1}=z^{\operatorname{deg}(D)} F(z)=F_{D}(z),
\end{aligned}
$$

and the product converges for $|z|<1$. Moreover, $F(z)=\zeta_{C}(s)$ for $z=q^{-s}$, and the function $F(z)$ will be the function $Z_{C}(t)$ from the Grothendieck cohomology theory 17 when we apply the substitution $t=q^{-s}$.

From $\S 2$, we know that

$$
\check{\pi}^{*}\left(\mathcal{L} f_{D}\right)=z^{\operatorname{deg} D} \frac{P(z)}{(1-z)(1-q z)},
$$

and as the target space in (24) we may take the space $\mathbb{C}_{++}\left[\mathbb{T}_{0}\right]$ of rational functions with possible first order poles at the points $z=1$ and $z=q^{-1}$. The image of the map $\tilde{\pi}^{*}$ can be identified with the space $P(z) \mathbb{C}_{++}\left[\mathbb{T}_{0}\right]$. This is easy to check by applying the map $\check{\pi}^{*}$ to standard functions.

The first main result of these notes is the following statement: the diagram

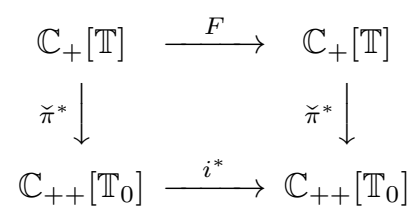

commutes. Here $i: \mathbb{T}_{0} \rightarrow \mathbb{T}_{0}$ is the involution $i(z)=q^{-1} z^{-1}$ of the torus $\mathbb{T}_{0}$. To obtain this commutativity, we can consider the maps for the standard functions and verify the property for them. Namely, we then have the diagram

$$
\begin{aligned}
& f_{D}=\bigotimes_{x} f_{x, D}=\bigotimes_{x} z_{x}^{v_{x}(D)}\left(1-z_{x}\right)^{-1} \stackrel{\breve{\pi}^{*}}{\longrightarrow} \quad F_{D}=z^{\operatorname{deg} D} Z_{C}(z) \\
& \mathrm{F} \downarrow \\
& \bigotimes_{x} q_{x}^{-\frac{1}{2} k_{x}+v_{x}(D)} f_{x, \mathrm{~K}-D} \quad \stackrel{\breve{\pi}^{*}}{\longrightarrow} q_{x}^{-\frac{1}{2} \operatorname{deg} \mathrm{K}+\operatorname{deg} D} z^{\operatorname{deg} \mathrm{K}-\operatorname{deg} D} Z_{C}(z) .
\end{aligned}
$$

Since $i^{*} F_{D}(z)=F_{D}\left(q^{-1} z^{-1}\right)$ we need to check the following claim:

$$
F_{D}\left(q^{-1} z^{-1}\right)=q^{-\frac{1}{2} \operatorname{deg} \mathrm{K}+\operatorname{deg} D} F_{\mathrm{K}-D}(z) .
$$

\footnotetext{
${ }^{17} \mathrm{~A}$ brief review of this theory can be found in Subsection 4.1.
} 
Indeed,

$$
\begin{aligned}
F_{D}\left(q^{-1} z^{-1}\right) & =q^{\operatorname{deg} D} z^{\operatorname{deg} D} P\left(q^{-1} z^{-1}\right)\left(1-q^{-1} z^{-1}\right)^{-1}\left(1-z^{-1}\right) \\
& \left.=q^{\operatorname{deg} D} z^{\operatorname{deg} D}\left[q^{-g} z^{-2 g}\right] P(z)\right] q z^{2}(1-q z)^{-1}(1-z)^{-1} \\
& =q^{-\frac{1}{2} \operatorname{deg} \mathrm{K}+\operatorname{deg} D} z^{-\operatorname{deg} \mathrm{K}+\operatorname{deg} D} P(z)(1-z)^{-1}(1-q z)^{-1} \\
& =q^{-\frac{1}{2} \operatorname{deg} \mathrm{K}+\operatorname{deg} D} F_{\mathrm{K}-D}(z),
\end{aligned}
$$

where $g=g(C)$, and we have used (25) and the relation for $i^{*} P$ that follows from the functional equation

$$
\zeta_{C}(s)=q^{g(C)-1} q^{-s(2 g-2)} \zeta_{C}(1-s)
$$

for $\zeta_{C}(s)=Z_{C}\left(q^{-s}\right)$ with $z=q^{-s}$.

In other words, in each local space the Fourier transform has additional factors that contribute to keeping the singularities of the functions at the prescribed points 1 and $q_{x}^{-1}$. The local Fourier transform does not correspond to any geometrical automorphism of the torus $\mathbb{T}_{x}$. The situation will drastically change when we go to the global space. In this case, the additional factors we have for each local Fourier transform will disappear when we multiply them over all the points of our curve $C$. Indeed, we have

$$
\left.\prod_{x} \frac{1-q_{x}^{-1} z_{x}^{-1}}{1-z_{x}}\right|_{\substack{z_{x}=z^{\operatorname{deg} x} \\ q_{x}=q^{\operatorname{deg} x}}}=Z_{C}(z) Z_{C}\left(q^{-1} z^{-1}\right)^{-1}=q^{\frac{1}{2} \operatorname{deg} \mathrm{K}} z^{\operatorname{deg} \mathrm{K}},
$$

and the last identity is exactly the functional equation.

We see that the functional equation is the symmetry with respect to the involution $i: z \mapsto q^{-1} z^{-1}$, once we identify the coordinate $z$ on the torus $\mathbb{T}_{0}$ with the parameter $t=q^{-s}$ from the Grothendieck theory. Then $i(z)=q^{-1} z^{-1}$ corresponds to the map $s \mapsto 1-s$ because $z=q^{-s}$.

The last remark we mention in this section concerns the function spaces and Fourier transforms on the connected component $\mathbb{T}_{0}$ of the torus $\mathbb{T}_{\text {Pic }}$. The Mellin transform connects the functions on the group $\Gamma_{C} / \Gamma_{0}=\mathbb{Z}$ with the functions on this torus. We have the following commutative diagram:

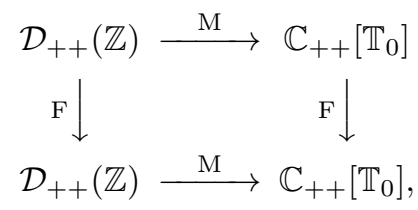

where

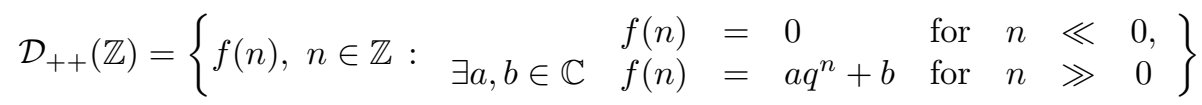

and

$$
(\mathrm{F} f)(n)=q^{n} f(-n) \quad \text { for } \quad n \in \mathbb{Z} \text { and } f \in \mathcal{D}(\mathbb{Z}) .
$$

By our construction, this space is exactly the image $\pi_{*}\left(\mathcal{D}_{+}\left(\Gamma_{C}\right)\right.$, where $\pi: \Gamma_{C} \rightarrow$ $\Gamma_{C} / \Gamma_{0}$ is the natural projection. We have then the commutative diagram

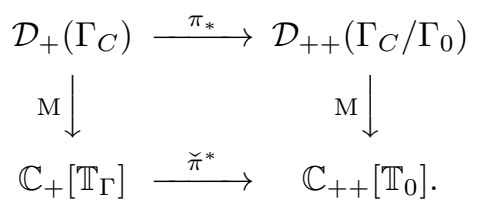


Up to now, we considered what happens to the functions on the torus $\mathbb{T}_{\Gamma}$ when they are restricted to the connected component $\mathbb{T}_{0}$ of unity of the torus $\mathbb{T}_{\text {Pic }}$. We have

$$
\mathbb{T}_{\text {Pic }}=\prod_{\chi \in \check{\Phi}} \chi \mathbb{T}_{0} .
$$

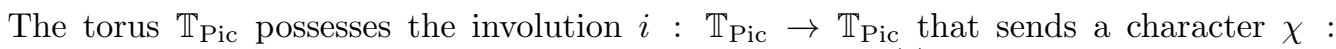
$\Gamma / \Gamma_{l} \rightarrow \mathbb{C}^{*}$ to the character $\breve{\chi}:=|\cdot| \chi^{-1}$ with $|a|=q^{-\operatorname{deg}(a)}$. This involution coincides with the previous involution $i$ on the torus $\mathbb{T}_{0}$. We claim that, again, there exists a commutative diagram

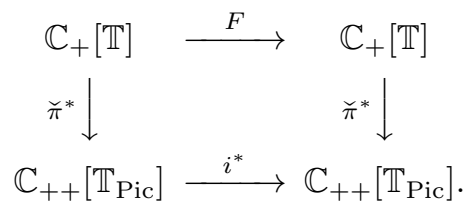

By the definition, the map $\check{\pi}: \mathbb{T}_{\text {Pic }} \rightarrow \mathbb{T}_{\Gamma}$ is given by the formula

$$
\check{\pi}(\chi)=\left(z_{x}, x \in C\right) \text { with } z_{x}=\chi\left(t_{x}\right),
$$

where $\chi \in \mathbb{T}_{\text {Pic }} \subset \operatorname{Hom}\left(\mathbb{A}^{*}, \mathbb{C}^{*}\right)$ and $t_{x}$ is a local parameter at the point $x \in C$, which gives rise to the element $\left(\ldots, 1, t_{x}, 1, \ldots\right)$ in $\mathbb{A}^{*}$. Then, formally, the inverse image of the standard function $\delta_{\geq 0}$ is equal to

$$
\prod_{x \in C}\left(1-\chi\left(t_{x}\right)\right)^{-1}
$$

For any $\chi \in \mathbb{T}_{\text {Pic }}$ we have the $L$-function $L_{C}(s, \chi)$ defined as the product

$$
L_{C}(s, \chi)=\prod_{x \in C}\left(1-\chi\left(t_{x}\right) q_{x}^{-s}\right)^{-1}=\prod_{x \in C}\left(1-\chi\left(t_{x}\right) t^{\operatorname{deg} x}\right)^{-1},
$$

where $t=q^{-s}$. If $\left.\chi\right|_{\Phi} \neq 1$, then this product converges for all $t$ and is a polynomial $P(t)$ in $t$ of degree $2 g$. It satisfies the functional equation

$$
P(t, \chi)=\chi((\omega)) q^{g-1} t^{2 g-2} P\left(q^{-1} t^{-1}, \chi^{-1}\right),
$$

where $\omega \in \Omega_{K}^{1}, \omega \neq 0$, is a rational differential form on $C$ and $(\omega)$ is the canonical class in $\operatorname{Pic}(C)$. This follows from the Tate-Iwasawa method, because this $L$-function is a particular case of the general $L$-functions from Subsection 2.3. Since our character $\chi$ is unramified, we may set $f=\delta_{\geq 0}$ in (13). See also Theorem 6 in W2, Chapter VII, no. 7].

It is easily seen that $P\left(q^{-1}, \chi^{-1}\right)=P\left(1,|\cdot| \chi^{-1}\right)$; consequently,

$$
P(1, \chi)=\chi((\omega)) q^{g-1} P\left(1,|\cdot| \chi^{-1}\right) .
$$

By definition, $\chi((\omega))=\prod_{x} \chi\left(t_{x}\right)^{\nu_{x}(\omega)}=\prod_{x} z_{x}^{k_{x}}$ and $q^{g-1}=\prod q_{x}^{\frac{1}{2} k_{x}}$. If we recall that $P(1, \chi)=\left.\breve{\pi}\left(\delta_{\geq 0}\right)\right|_{\chi \mathbb{T}_{0}}$, for this inverse image we get the following identity:

$$
\left.\check{\pi}\left(\delta_{\geq 0}\right)\right|_{\chi \mathbb{T}_{0}}=\left.\prod_{x} z_{x}^{k_{x}} q_{x}^{\frac{1}{2} k_{x}} \check{\pi}\left(\delta_{\geq 0}\right)\right|_{\breve{\chi} \mathbb{T}_{0}} .
$$

The same relation is valid for the inverse images of the standard functions $f_{D}$ for any divisor $D$. Now we can repeat all the computations made above for the component $\mathbb{T}_{0}$ and get diagram (30).

Finally, we say a few words about the Weil problem for the functional $\Delta_{\chi}$ in this new setting. The local component of the integral (13) will be an integral over $K_{x}^{*}$, which is equal to the arithmetical progression for the function $\left(1-z_{x}\right)^{-1}$ and converges for 
$\left|z_{x}\right|<1$. Then the functional $\Delta_{\chi}\left(f_{x}\right)$ is well defined for all $z_{x} \neq 1$ by the evaluation formula

$$
\Delta_{\chi}: \mathbb{C}_{+}\left[\mathbb{T}_{x}\right] \rightarrow \mathbb{C}, \quad \Delta_{\chi}\left(f_{x}\right)=f_{x}\left(z_{x}\right),
$$

where the character $\chi$ corresponds to the complex number $z_{x} \in \mathbb{T}_{x}$. This will provide a solution of the Weil problem for functions on the local tori. Indeed, the structure of a $\mathcal{D}(\mathbb{Z})$-module on the space $\mathcal{D}_{+}(\mathbb{Z})$, defined by convolution (see Subsection 2.3), goes to the structure of a $\mathbb{C}\left[\mathbb{T}_{x}\right]$-module on $\mathbb{C}_{+}\left[\mathbb{T}_{x}\right]$, defined by multiplication. On $\mathbb{C}\left[\mathbb{T}_{x}\right]$ we have the functional $\Delta_{\chi}$ defined by the evaluation formula for all $\chi$, and we are looking for $\Delta_{\chi}$ on $\mathbb{C}_{+}\left[\mathbb{T}_{x}\right]$ such that

$$
\Delta_{\chi}(f \cdot g)=f\left(z_{x}\right) \Delta_{\chi}(g) \text { for all } f \in \mathbb{C}\left[\mathbb{T}_{x}\right] \text { and } g \in \mathbb{C}_{+}\left[\mathbb{T}_{x}\right]
$$

see (14). The solution for $\chi \neq 1$ was given above. If $\chi=1$, so that $z_{x}=1$, we set

$$
\Delta_{\chi}\left(f_{x}\right)=\operatorname{res}_{(1)}\left(f\left(z_{x}\right) \omega\right)
$$

where $\omega=d z_{x} / z_{x}$. Concerning the global case, see Subsection 3.5 below.

3.4. The Poisson formula and residues. The involution $i: \mathbb{T}_{0} \rightarrow \mathbb{T}_{0}$ extends uniquely to a map on the projective compactification $\overline{\mathbb{T}}_{0}=\mathbb{P}^{1}$ of the torus $\mathbb{T}_{0}=\mathbb{C}^{*}$. Let $\omega=d z / z$. Then

$$
i^{*}(\omega)=d\left(q^{-1} z^{-1}\right) / q^{-1} z^{-1}=d z^{-1} / z^{-1}=-\omega .
$$

Let $g \in \mathbb{C}_{++}\left[\mathbb{T}_{0}\right]$. The divisor of poles has the support

$$
\operatorname{Supp}(g)_{\infty} \subset\{z=0\} \cup\left\{z=q^{-1}\right\} \cup\{z=1\} \cup\{z=\infty\} .
$$

Thus,

$$
\operatorname{res}_{(0)}(g \omega)+\operatorname{res}_{\left(q^{-1}\right)}(g \omega)+\operatorname{res}_{(1)}(g \omega)+\operatorname{res}_{(\infty)}(g \omega)=0 .
$$

Since $\operatorname{res}_{P}(\eta)=\operatorname{res}_{i(P)}\left(i^{*} \eta\right)$ for any differential form $\eta$ and any point $P$, we have

$$
\operatorname{res}_{(0)}(g \omega)+\operatorname{res}_{(1)}(g \omega)=\operatorname{res}_{(0)}\left(i^{*} g \cdot \omega\right)+\operatorname{res}_{(1)}\left(i^{*} g \cdot \omega\right) .
$$

The torus $\mathbb{T}_{0}$ is a connected component of $\mathbb{T}_{\text {Pic }}$, and we have

$$
\mathbb{T}_{\text {Pic }}=\coprod_{\chi \in \check{\Phi}} \chi \mathbb{T}_{0} \subset \overline{\mathbb{T}}_{\text {Pic }}
$$

where the boundary of the compactification $\overline{\mathbb{T}}_{\text {Pic }}$ has the following structure:

$$
\partial \overline{\mathbb{T}}_{\text {Pic }}=\coprod_{\chi \in \check{\Phi}}\left\{0_{\chi}\right\} \coprod_{\chi \in \check{\Phi}}\left\{\infty_{\chi}\right\} .
$$

Each component $\chi \mathbb{T}_{0}$ is isomorphic to $\mathbb{C}^{*}$ with a coordinate $z$, and the points $0_{\chi}$ and $\infty_{\chi}$ are defined as $z=0$ and $z=\infty$ respectively. The form $\omega$ on $\mathbb{T}_{0}$ is invariant under translations, thus giving rise to a canonical invariant form $\omega$ on $\mathbb{T}_{\text {Pic }}$.

Now, we introduce the function space $\mathbb{C}_{++}\left[\mathbb{T}_{\text {Pic }}\right]$ on $\mathbb{T}_{\text {Pic }}$ as the direct sum of the space $\mathbb{C}_{++}\left[\mathbb{T}_{0}\right]$ and the spaces $\mathbb{C}\left[\chi \mathbb{T}_{0}\right]$, where $\chi \in \check{\Phi}$ and $\chi \neq 1$.

We are ready obtain our second main result. Let $f \in \mathcal{D}_{+}(\Gamma)$. Then we have a sequence of transformations

$$
f \mapsto \mathrm{M} f \in \mathbb{C}_{+}[\mathbb{T}]_{\Gamma} \mapsto \tilde{f}:=\check{\pi}^{*} \mathrm{M} f \in \mathbb{C}_{++}\left[\mathbb{T}_{\text {Pic }}\right],
$$

where the map $\pi$ is again the natural projection onto the larger group $\Gamma / \Gamma_{l}$ (see Subsection 3.3).

We claim that the identity (where the residues at $z=1$ are performed on the torus $\mathbb{T}_{0}$ )

$$
\sum_{\chi \in \breve{\Phi}} \operatorname{res}_{\left(0_{\chi}\right)}(\tilde{f} \omega)+\operatorname{res}_{(1)}(\tilde{f} \omega)=\sum_{\chi \in \check{\Phi}} \operatorname{res}_{\left(0_{\chi}\right)}\left(i^{*} \tilde{f} \cdot \omega\right)+\operatorname{res}_{(1)}\left(i^{*} \tilde{f} \cdot \omega\right)
$$


is equivalent to the Poisson formula (21) for the function $f$. Since $\# \check{\Phi}=\# \Phi$, this is implied from the following facts:

(1) $i^{*}(\tilde{f})=\widetilde{\mathrm{Ff}}$;

(2) $\sum_{\chi \in \check{\Phi}} \operatorname{res}_{\left(0_{\chi}\right)}(\tilde{f} \omega)=\# \check{\Phi} \delta_{\Gamma_{l}}(f)$;

(3) $\operatorname{res}_{(1)}(\tilde{f} \omega)=\# \Phi /(q-1) \delta_{(0)}(f)$.

The first property follows from the commutative diagrams (26) and (30) considered above.

Concerning the second property, note that for $f \in \mathcal{D}_{+}(\Gamma)$ we have $\delta_{\Gamma_{l}}(f)=\left(\pi_{*} f\right)(0)$. We want to go from the group $\Gamma / \Gamma_{l}$ to the dual torus $\mathbb{T}_{\text {Pic }}$.

In general, for a discrete group $G$ and a function $\varphi$ on $G$ we have $(\mathrm{M} \varphi)(\chi)=$ $\sum_{g \in G} \varphi(g) \chi(g)$, where $\chi \in \mathbb{T}$ and $\mathbb{T}$ is the dual group. Then,

$$
\varphi(0)=\int_{\mathbb{T}}(\mathrm{M} \varphi)
$$

where the integral can be defined as

$$
\int_{\mathbb{T}} \psi=(\# \mathbb{T})^{-1} \sum_{\chi \in \mathbb{T}} \psi(\chi)
$$

whenever the group $\mathbb{T}$ is finite, and

$$
\int_{\mathbb{T}} \psi=\operatorname{res}_{(0)}(\psi d z / z)
$$

if $\mathbb{T} \cong \mathbb{C}^{*} 18$ This immediately gives the property in question.

The third property. Let $f=f_{D}=\bigotimes_{x \in C} \delta_{\left(\geq n_{x}\right)}=\bigotimes_{x \in C} f_{x}$ for a divisor $D=$ $\sum_{x} n_{x} \cdot x$. Then $\delta_{(0)}(f)=\prod_{x} \delta_{(0)}\left(f_{x}\right)=\prod_{x} \lim _{n \rightarrow+\infty} f_{x}(n)=1$. As we saw above, $\widetilde{f}=z^{\operatorname{deg} D} Z(z)$, the computation of the residue of the zeta-function at the point $s=0$, i.e., $z=1$ (see Subsection 2.5) shows that $\operatorname{res}_{(1)}(\tilde{f} \omega)=\# \operatorname{Pic}^{0}(C)\left(\mathbb{F}_{q}\right) /(q-1)$. The general case follows by linearity, because any function $f$ is a finite $\operatorname{sum} \sum_{D} c_{D} f_{D}$.

Scholium 2. In the étale cohomology theory we have $\zeta_{C}(s)=Z_{C}(t)$ with $t=q^{-s}$, and the parameter $t$ appears in a purely formal manner (see Subsection 4.1 below). Our torus coordinate $z$ does appear in a canonical way as a coordinate on the connected component of the torus dual to the group $\Gamma / \Gamma_{0}=\operatorname{Pic}(C)$. We can say that $t=z$, and thus, the function $Z_{C}(t)$ is defined naturally on $\mathbb{P}^{1} \supset \mathbb{C}^{*}$, rather than only on the torus $\mathbb{C}^{*}$.

Also, we can rewrite the more general Poisson formula in which the shifted $\delta$-function appears. For a given $g \in \mathbb{A}^{*}$, the analog of the Poisson formula (22) with $\delta_{g K}$ will be the following relation for residues:

$$
\operatorname{res}_{(0)}\left(z^{n} \tilde{f} \omega\right)+\operatorname{res}_{(1)}(\tilde{f} \omega)=\operatorname{res}_{(0)}\left(z^{-n} i^{*} \tilde{f} \cdot \omega\right)+\operatorname{res}_{(1)}\left(i^{*} \tilde{f} \cdot \omega\right),
$$

where $n=\operatorname{deg} \gamma$ and $\gamma$ is the image of $g$ in the group $\Gamma_{C}=\mathbb{A}^{*} / \mathcal{O}^{*} 19$

3.5. Explicit formulas. The procedure we have carried out here is very similar to the way in which the so-called explicit formulas are deduced in analytic number theory. We present the corresponding argument in our language and compare it with the traditional exposition in the next section.

\footnotetext{
${ }^{18}$ Compare with the maps $\alpha^{*}$ and $\beta_{*}$ in diagram (2).

${ }^{19}$ Here, for simplicity, we have assumed that $\Phi=(1)$, i.e., $\Gamma_{0}=\Gamma_{l}$. Then, in order to obtain this formula, we need a more general relation than above, specifically, $\varphi(g)=\int_{\mathbb{T}} \chi(g)(M \varphi)(\chi) d \chi$.
} 
Let $P(z)(1-z)^{-1}(1-q z)^{-1}=Z(z)=\zeta_{C}(s)$ for $z=q^{-s}$. Then $\omega=d Z / Z$ is a logarithmic differential form on the projective line $\mathbb{P}^{1}$, and

$$
\operatorname{Supp}(\omega)_{\infty} \subset\{z=0\} \cup\left\{z=q^{-1}\right\} \cup \bigcup_{\lambda}\left\{z=\lambda^{-1}\right\} \cup\{z=1\} \cup\{z=\infty\},
$$

where $P(z)=\prod_{\lambda}(1-\lambda z)$ is the spectral decomposition for the characteristic polynomial of the Frobenius automorphism. Suppose $h$ belongs to $\mathbb{C}\left[\mathbb{T}_{0}\right]$ and comes from a function $f \in \mathcal{D}(\mathbb{Z})$ via the Mellin transform (see (23) $)$. Taking the sum of residues as above, we obtain

$$
\operatorname{res}_{(0)}(h \omega)+\operatorname{res}_{\left(q^{-1}\right)}(h \omega)+\sum_{\lambda} \operatorname{res}_{\left(\lambda^{-1}\right)}(h \omega)+\operatorname{res}_{(1)}(h \omega)+\operatorname{res}_{(\infty)}(h \omega)=0 .
$$

We want to compute all the members of this sum:

(1) $\operatorname{res}_{\left(q^{-1}\right)}(h \omega)=-h\left(q^{-1}\right)$;

(2) $\operatorname{res}_{\left(\lambda^{-1}\right)}(h \omega)=h\left(\lambda^{-1}\right)$;

(3) $\operatorname{res}_{(1)}(h \omega)=-h(1)$;

(4) $\operatorname{res}_{(\infty)}(h \omega)=\operatorname{res}_{(0)}(\widetilde{\mathrm{F} f} \omega)+(2-2 g(C)) \operatorname{res}_{(0)}(\widetilde{\mathrm{F} f} d z / z)$;

(5) $\operatorname{res}_{(0)}(h \omega)=-\sum_{x \in C, n>1}(\operatorname{deg} x) f(-n \operatorname{deg}(x))$.

The first three properties follow directly from computation of logarithmic residues and our knowledge of the behavior of $Z(z)$ at the points $z=q^{-1}, z=\lambda^{-1}, z=1$.

To get the fourth property, we apply the involution $i$, which transforms the point $z=\infty$ to the point $z=0$, and use the invariance of the residue. Next, the functional equation (27) implies that $i^{*}(\omega)=(2-2 g(C)) d z / z+\omega$ and our diagram (26) shows that $i^{*} h=\widetilde{\mathrm{F} f}$ if the original function $h$ was produced from $f$.

To get the fifth property, we start with the Euler product $Z(z)=\prod_{x}\left(1-z^{\operatorname{deg} x}\right)^{-1}$. In a neighborhood of the point $z=0$, we have

$$
\omega=d \ln Z=-\sum_{x \in C, n \geq 1}(\operatorname{deg} x) z^{(n \operatorname{deg} x)} d z / z .
$$

If $h(z)=\sum a_{m} z^{m}$, we arrive at the finite sum

$$
\operatorname{res}_{(0)}(h \omega)=-\sum_{x \in C, n \geq 1}(\operatorname{deg} x) a_{-n \operatorname{deg} x},
$$

where $n \operatorname{deg} x \leq-\nu_{(0)}(h)$.

Since we have started with a function $f$ defined on the group $\Gamma / \Gamma_{0}=\mathbb{Z}$, we have $a_{m}=f(m), m \in \Gamma / \Gamma_{0}$, and the final formula will be

$$
\begin{aligned}
h\left(q^{-1}\right) & -\sum_{P\left(\lambda^{-1}\right)=0} h\left(\lambda^{-1}\right)+h(1) \\
& =(2-2 g(C)) \mathrm{F} f(0)-\sum_{x \in C, n \geq 1}(\operatorname{deg} x)[f(-n \operatorname{deg} x)+\mathrm{F} f(-n \operatorname{deg} x)] \\
& =(2-2 g(C)) f(0)-\sum_{x \in C, n \geq 1}(\operatorname{deg} x)\left[f(-n \operatorname{deg} x)+q^{-n \operatorname{deg} x} f(n \operatorname{deg} x)\right] .
\end{aligned}
$$

Here, we have replaced $\mathrm{F} f(0)$ by $f(0)$ and $\mathrm{F} f(n)$ by $q^{n} f(-n)$.

This is exactly the explicit formula for the case of function fields (see [W3, C] and compare with the case of number fields in [L1, VK]). The first explicit formula was found by Riemann. It relates the distribution of prime numbers to a sum over critical zeros of the zeta function. Later, de la Vallée-Poussin and Hadamard added some arguments from complex analysis; after that, this formula led to the prime number theorem. In our case, the closed points $x$ replace the prime numbers and the eigenvalues $\lambda$ replace the 
critical zeros of the zeta function. The corresponding asymptotic law is also valid and can be stated as follows:

$$
\#\{x \in C: N(x)<N\} \sim \frac{N}{\ln N} \quad \text { as } \quad N \rightarrow \infty,
$$

where $N(x)=q_{x}=q^{\operatorname{deg} x}=\# k(x)$.

To obtain this from the explicit formula (26), one chooses for $f$ the test function $f=\delta_{\geq N}$. The de la Vallée-Poussin and Hadamard argument was to show that there are no zeros of the zeta-function on the boundary $\operatorname{Re}(s)=1$ of the critical strip. In our case, this is equivalent to the inequality $|\lambda|<q$ (the critical strip will be $q^{-1} \leq z \leq 1$ ), which is much weaker than the Riemann conjecture $|\lambda|=q^{-1 / 2}$ (Weil's theorem) .

We also note that, by a simple computation, the asymptotic law is equivalent to the following asymptotic behavior for the number of rational points on the curve $X$ :

$$
\frac{X\left(\mathbb{F}_{q^{n}}\right)}{q^{n}} \rightarrow 1 \text { as } n \rightarrow \infty
$$

\section{$\S 4$. Trace formula and the Artin Representation}

4.1. Zeta functions and étale cohomology. Let $X$ be a scheme of finite type over $\mathbb{Z}$. We denote by $|X|$ the set of closed points of $X$. For every $x \in|X|$, the residue field $k(x)$ is a finite field. The zeta function of $X$ is defined by

$$
\zeta(X, s):=\prod_{x \in|X|}\left(1-(\# k(x))^{-s}\right)^{-1}, \quad s \in \mathbb{C} .
$$

This expression is called the Euler product for the zeta-function. The Euler product converges for $\operatorname{Re}(s)>\operatorname{dim}(X)$.

The first examples are the following:

1) $\zeta\left(\operatorname{Spec}\left(\mathbb{F}_{q}\right), s\right)=\left(1-q^{-s}\right)^{-1}$;

2) $\zeta(\operatorname{Spec}(\mathbb{Z}), s)=\zeta(s)$, the Riemann zeta function;

3) Let $X / \mathbb{F}_{q}$ be a scheme over a finite field. Then

$$
\zeta(X, s)=\exp \left(\sum_{m=1}^{\infty} \# X\left(\mathbb{F}_{q^{m}}\right) \frac{q^{-m s}}{m}\right)
$$

in the ring of formal power series, and the series is absolutely convergent for $\operatorname{Re}(s)>\operatorname{dim}(X)$.

To prove 3 ), we should take the logarithmic derivative of both sides in the ring of formal power series and then use the relation

$$
\# X\left(\mathbb{F}_{q^{m}}\right)=\sum_{l \mid m} a_{l} l, \quad m=1,2, \ldots,
$$

where $a_{l}$ denotes the number of closed points $x \in X$ for which $\# k(x)=q^{l}$. This number is finite. Also, the absolute convergence of the infinite product and of the power series are equivalent.

Now if $X=\mathbb{A}_{\mathbb{F}_{q}}^{n}$, we see that $\zeta(X, s)=\exp \left(\sum_{m=1}^{\infty} q^{m(n-s)} / m\right)$, and the sum on the right-hand side is absolutely convergent for $\left|q^{n-s}\right|<1$, i.e., for $\operatorname{Re}(s)>n=\operatorname{dim}(X)$. Furthermore, we see that

4) $\zeta\left(\mathbb{A}_{\mathbb{F}_{q}}^{n}, s\right)=\left(1-q^{n-s}\right)^{-1}$.

In the general case, the convergence of the Euler product can easily be reduced to this case. For projective varieties over $\mathbb{F}_{q}$, the main tool for the study of the zeta (and also $L-)$ functions is étale cohomology, or more precisely, $l$-adic cohomology for some prime number $l$ such that $(l, q)=1$. Let us fix such an $l$ and a ground field $k$. 
For every projective variety (actually, for much more general schemes) $X / k$, there exists a family

$$
H^{i}\left(X, \mathbb{Q}_{l}\right), \quad i=0,1, \ldots,
$$

of $\mathbb{Q}_{l}$-vector spaces called the $i$ th $l$-adic cohomology groups with values in $\mathbb{Q}_{l}$. The groups $H^{i}\left(X, \mathbb{Q}_{l}\right)$ are finite-dimensional vector spaces over $\mathbb{Q}_{l}$ and are trivial for $i>2 \operatorname{dim}(X)$ if the ground field $k$ is algebraically closed.

The cohomology group $H^{i}\left(X, \mathbb{Q}_{l}\right)$ is contravariant with respect to morphisms of varieties, and we have the usual nondegenerate cup product pairings on them; see the standard textbooks.

When $k=\mathbb{C}$, these groups coincide with the groups $H^{i}(X, \mathbb{Q}) \otimes_{\mathbb{Q}} \mathbb{Q}_{l}$, where $H^{i}(X, \mathbb{Q})$ are the usual cohomology groups of the topological space $X(\mathbb{C})$ (for the classical topology) and many properties of the topological cohomology groups remain true in the $l$-adic setting. In particular, there is the Lefschetz fixed point formula for a morphism $f$ : $X \rightarrow X$ of projective varieties defined over an algebraically closed field. We have

$$
\sum_{i}(-1)^{i} \operatorname{Tr}\left(f: H^{i}\left(X, \mathbb{Q}_{l}\right) \rightarrow H^{i}\left(X, \mathbb{Q}_{l}\right)\right)=\sum_{x \in X} \#\left(\Gamma_{f} \cap \Delta\right)_{(x, x)},
$$

under the assumption that the graph $\Gamma_{f}$ of the morphism and the diagonal $\Delta \subset X \times X$ are in general position in $X \times X$. If we assume that the subvariety $\Gamma_{f}$ intersects $\Delta$ transversally, then all the multiplicities at the fixed points $x \in X$ of $f$ are equal to 1 , and we can conclude that the formula counts the number of the fixed points:

$$
\sum_{i}(-1)^{i} \operatorname{Tr}\left(f: H^{i}\left(X, \mathbb{Q}_{l}\right) \rightarrow H^{i}\left(X, \mathbb{Q}_{l}\right)\right)=\sum_{x \in X: f(x)=x} 1 .
$$

In order to show how this formula can be applied to the study of zeta-functions, we need the Frobenius automorphism. It is well defined as an automorphism $\mathrm{Fr}: \bar{X} \rightarrow \bar{X}$ of the scheme $\bar{X}=X \bigotimes_{\mathbb{F}_{q}} \overline{\mathbb{F}}_{q}$ over $\overline{\mathbb{F}}_{q}$ for any scheme $X$ over a finite field $\mathbb{F}_{q}$. For the scheme $X$ over $\mathbb{F}_{q}$, we define $F r_{X}$ as identity on the topological space $X$ and as the $q$ th power on the structure sheaf $\mathcal{O}_{X}$. On the scheme $\bar{X}$, the Frobenius morphism $F r$ is $F r_{X} \otimes 1_{\overline{\mathbb{F}_{q}}}$. If the scheme $X$ is embedded into a projective space with coordinates $\left(x_{0}: x_{1}: \ldots: x_{n}\right)$, then we have an embedding of the scheme $\bar{X}$ into the same projective space. Then the Frobenius morphism will be the restriction to $\bar{X}$ of the map $\left(x_{0}: x_{1}: \ldots: x_{n}\right) \mapsto\left(x_{0}^{q}: x_{1}^{q}: \ldots: x_{n}^{q}\right)$. The following properties occur.

1. $\bar{X}^{F r^{n}}=X\left(\mathbb{F}_{q^{n}}\right)$, where $\bar{X}^{F r^{n}}$ denotes the subset of closed points of $\bar{X}$ that are fixed by $\mathrm{Fr}^{n}$.

2. The set of closed points $x$ of $X$ can be identified with the set of orbits of $F r$ acting on the set of closed points of $\bar{X}$. The integer $\operatorname{deg}(x)=\operatorname{dim}_{\mathbb{F}_{q}} k(x)$ is equal to the number of elements in the orbit corresponding to $x$.

3. $\# \bar{X}^{F r^{n}}=\sum_{x \in X, \# k(x) \mid n} \operatorname{deg}(x)$.

In our case of projective varieties, we have an induced action

$$
F r^{*}: H^{i}\left(\bar{X}, \mathbb{Q}_{l}\right) \rightarrow H^{i}\left(\bar{X}, \mathbb{Q}_{l}\right) \text {. }
$$

The differential of $F r$ is the zero map. This implies that the graph of $F r$ intersects the diagonal transversally, and now the Lefschetz fixed point formula for $l$-adic cohomology states that, for $n=1,2, \ldots$,

$$
\# \bar{X}^{F r^{n}}=\sum_{0 \leq i \leq 2 \operatorname{dim}(X)}(-1)^{i} \operatorname{Tr}\left(\left(F r^{*}\right)^{n}: H^{i}\left(\bar{X}, \mathbb{Q}_{l}\right) \rightarrow H^{i}\left(\bar{X}, \mathbb{Q}_{l}\right)\right)
$$


For an arbitrary linear operator $F$ acting on a finite-dimensional vector space $V$, we have the following relation in the ring of formal power series 20

$$
t \frac{d}{d t} \log (\operatorname{det}(1-F t))^{-1}=\sum_{n=1}^{\infty} \operatorname{Tr}\left(F^{n}\right) t^{n} .
$$

Using relation (33), it makes sense to set $\zeta(X, s)=Z(X, t)$ with $t=q^{-s}$. We get

$$
\begin{aligned}
t \frac{d}{d t} \log Z(X, t) & =\sum_{n \geq 1} \# X\left(\mathbb{F}_{q^{n}}\right) t^{n} \\
& =\sum_{i \geq 0}(-1)^{i} \sum_{n \geq 1} \operatorname{Tr}\left(\left(F r^{*}\right)^{n}: H^{i}\left(\bar{X}, \mathbb{Q}_{l}\right) \rightarrow H^{i}\left(\bar{X}, \mathbb{Q}_{l}\right)\right) \\
& =\sum_{i \geq 0}(-1)^{i} t \frac{d}{d t} \log \left(\left(\operatorname{det}\left(1-F r^{*} t \mid H^{i}\left(\bar{X}, \mathbb{Q}_{l}\right)\right)\right)^{-1}\right)
\end{aligned}
$$

Therefore,

$$
Z(X, t)=\prod_{0 \leq i \leq 2 \operatorname{dim}(X)}\left(\operatorname{det}\left(1-F r^{*} t \mid H^{i}\left(\bar{X}, \mathbb{Q}_{l}\right)\right)\right)^{(-1)^{i+1}}=P(t) / Q(t)
$$

This provides a description of the zeta function in terms of $l$-adic cohomology, and we see immediately that $\zeta(X, s)$ is a rational function in $q^{-s}$ because the spaces $H^{i}\left(\bar{X}, \mathbb{Q}_{l}\right)$ are finite-dimensional.

In the case of an algebraic curve $C$, we have $P(t)=\operatorname{det}\left(1-F r^{*} t \mid H^{1}\left(\bar{C}, \mathbb{Q}_{l}\right)\right)($ a polynomial of degree $g(C))$ and $Q(t)=\operatorname{det}\left(1-F r^{*} t \mid H^{0}\left(\bar{C}, \mathbb{Q}_{l}\right)\right) \operatorname{det}\left(1-F r^{*} t \mid H^{2}\left(\bar{C}, \mathbb{Q}_{l}\right)\right)=$ $(1-t)(1-q t)$, as we have already found above.

The last remark is that the Poincaré duality is well defined in $l$-adic cohomology and implies the functional equation for zeta functions.

4.2. Local Artin representation. Let $K$ be a local field (of dimension 1), i.e., the field of fractions of a complete discrete valuation $\operatorname{ring} \mathcal{O}$, and let $L / K$ be a finite normal extension. Then $K \supset \mathcal{O}_{K} \supset \mathfrak{m}_{K}$, where $\mathfrak{m}$ is the maximal ideal of $\mathcal{O}_{K}$ and $k$ the residue field of $K$. Let $l$ be the residue field of $L$. Let $g \in \operatorname{Gal}(L / K)$. We set:

$$
i_{G}(g)=\text { the maximal integer } i \text { such that } g \text { acts trivially on } \mathcal{O}_{L} / \mathfrak{m}_{L}^{i+1} \text {. }
$$

We have $i \geq-1$ and $i_{G}(e)=\infty$. The function $i_{G}$ gives rise to a filtration $\left\{G_{i}\right\}$ of the group $\operatorname{Gal}(L / K)$. An extension is unramified if and only if $G_{0}=\{e\}$, i.e., if $i_{G}(g)=-1$ for all $g \neq e$.

If $g \in G_{0}$, then we can redefine the function $i_{G}$ :

$$
i_{G}(g)=\nu_{L}(g(t)-t), \quad g \neq e,
$$

where $\nu_{L}$ is the discrete valuation of the field $L$ and $t$ is a generator of the maximal ideal $\mathfrak{m}_{L}$.

The basic observation is that if we introduce the new function $a_{G}$ on $G$ by

$$
a_{G}(g)= \begin{cases}-i_{G}(g)[l: k] & \text { if } g \neq e \\ \sum_{g \neq e} i_{G}(g)[l: k] & \text { if } g=e\end{cases}
$$

then this function will be a character of some (finite-dimensional) representation of the group $G$ over $\mathbb{C}$. This fact can be proved in quite a nondirect way, and it is still an

\footnotetext{
${ }^{20}$ For the proof, the ground field can be assumed algebraically closed. Then the 1-dimensional case can be checked directly, and the general case reduces to it by the additivity of both sides of the formula with respect to short exact sequences of vector spaces $V$.
} 
open problem to define this representation, called the Artin representation, in an explicit manner (see [S2]).

Suppose the residue field of $K$ is a finite field $\mathbb{F}_{q}$ and the field $K$ is a field of equal characteristics. There is class field theory describing the Galois group of the maximal Abelian extension of $K$; see [A, W2, S2]. We have the commutative diagram

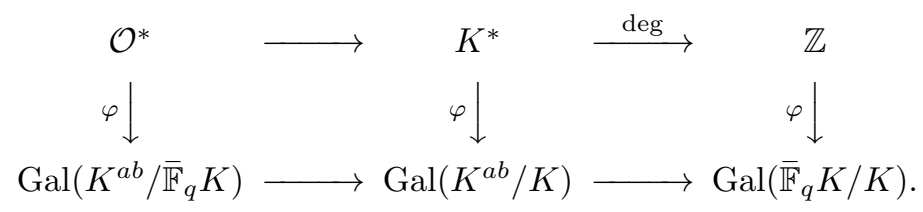

The system of groups $G^{i}:=G_{\psi(i)}$, where $\psi: \mathbb{Z} \rightarrow \mathbb{Z}$ is the Herbrand function (see $\mathrm{A}$, W3, S2 ) will be consistent in the tower of all finite normal extensions of the field $K$. Thus, we can define the Artin representations of infinite extensions, including the maximal Abelian extension. The reciprocity map $\varphi$ has the following property:

$$
\varphi\left(1+\mathfrak{m}^{i}\right)=\operatorname{Gal}\left(K^{a b} / K\right)_{\psi(i)} .
$$

This means that the Artin character can also be defined on the group $K^{*}$. Then the corresponding function $a_{K}$ can be viewed as a distribution on the locally compact group $K^{*}$ and $a_{K} \in \mathcal{D}^{\prime}\left(K^{*}\right)$. The definition is rather subtle but still very explicit:

$$
a_{K}(f)=\int_{\mathcal{O}^{*}} \frac{f(g)-f(1)}{|1-g|} d^{*} g+\int_{K^{*}-\mathcal{O}^{*}} \frac{f(g)}{|1-g|} d^{*} g, \quad f \in \mathcal{D}\left(K^{*}\right) .
$$

The integral without subtraction of $f(1)$ can be divergent if $f(1) \neq 0$, and the given formula contains a regularization required in order to define the integral for any function belonging to the space $\mathcal{D}\left(K^{*}\right)$ (see [W3, C]).

Assume that the function $f$ is invariant under $\mathcal{O}^{*}$. Then the first term is zero, $K^{*}=$ $\coprod_{n} t^{n} \mathcal{O}^{*}$, and for the full character we get

$$
\begin{aligned}
a_{K}(f) & =\int_{K^{*}-\mathcal{O}^{*}} \frac{f(g)}{|1-g|} d^{*} g \\
& =\sum_{n \geq 1} \int_{t^{n} \mathcal{O}^{*}} f\left(t^{n}\right)|1-g|^{-1} d^{*} g+\sum_{n \leq-1} \int_{t^{n} \mathcal{O}^{*}} f\left(t^{n}\right)|1-g|^{-1} d^{*} g \\
& =\sum_{n \geq 1} f\left(t^{n}\right) \operatorname{vol}\left(\mathcal{O}^{*}\right)+\sum_{n \leq-1} \int_{t^{n} \mathcal{O}^{*}} f\left(t^{n}\right) q^{-n} \operatorname{vol}\left(\mathcal{O}^{*}\right) \\
& =\operatorname{vol}\left(\mathcal{O}^{*}\right) \sum_{n \geq 1}\left[f\left(t^{n}\right)+f\left(t^{-n}\right) q^{n}\right] .
\end{aligned}
$$

Here, we have used the fact that $|1-g|=1$ if $|g|<1$ and $|1-g|=|g| \cdot\left|1-g^{-1}\right|=|g|$ if $|g|>1$.

We return to the global case. Let $X$ be an algebraic curve (as usual irreducible, projective, and smooth) defined over a finite field $\mathbb{F}_{q}$, and let $G$ be a finite group of automorphisms of $X$. Also, we have the curve $C=X / G$ and a finite covering map $\varphi: X \rightarrow C$. If $L=\mathbb{F}_{q}(X)$ and $K=\mathbb{F}_{q}(C)$ are the fields of rational functions on the curves, then $G=\operatorname{Gal}(L / K)$ and for any point $y \in X$ we have the decomposition group $D_{y} \subset G$, defined by $D_{y}=\{g \in G: g(y)=y\}$. If $x=\varphi(y)$, there is a finite normal extension of the local fields $L_{y} / K_{x}$ with the Galois group $D_{y}$. Thus, on the groups $D_{y}$ we have the functions $i_{G}$ and $a_{G}$, which we denote by $i_{y}$ and $a_{y}$.

We can show that

$$
i_{y}(g)=\nu_{y}(g(t)-t)=\left(\Gamma_{g} \cdot \Delta\right)_{(y, y)}
$$


where $\Gamma_{g}$ is the graph of the automorphism $g$ in the surface $X \times X$ and $\Delta$ is the diagonal. The first identity has already been mentioned. The second follows from the definition of the intersection multiplicity of $\Gamma_{g}$ with $\Delta$ at the point $(y, y)$. Indeed, in local coordinates $u, t$, an equation for $\Gamma_{g}$ is given by $f_{1}=g(u)-t$ near the point $(y, y)$, and an equation for $\Delta$ by $f_{2}=u-t$. By definition, $\left(\Gamma_{g} \cdot \Delta\right)_{(y, y)}=\operatorname{dim} \mathcal{O}_{(y, y)} /\left(f_{1}, f_{2}\right)$ and $\operatorname{dim} \mathcal{O}_{(y, y)} /\left(f_{1}, f_{2}\right)=$ $\operatorname{dim} \mathcal{O}_{y} /(g(u)-u)$.

For all $y$ lying over a given $x \in C$, the functions $a_{y}$ can be extended by zero to the entire group $G$, and then we can set

$$
a_{x}:=\sum_{y \mapsto x} a_{y} .
$$

For any point $y$ lying over $x$, the character $a_{x}$ is induced by $a_{y}$. The representation $\pi_{x}$ of the Galois group $G$ defined by the character $a_{x}$ will be called the Artin representation at the point $x \in C$.

We arrive at the final point of this construction, an application of the Lefschetz trace formula:

$$
\operatorname{Tr}(g) \mid H^{\bullet}\left(\bar{X}, \mathbb{Q}_{l}\right)=(2-2 g(C)) \# G \delta_{G}(g)-\sum_{x \in C} a_{x}(g),
$$

where $\delta_{G}(g)=0$ for $g \neq e$ and $\delta_{G}(e)=1$.

Proof. For $g \neq e$ this is exactly the Lefschetz trace formula (35) applied to the extension of the automorphism $g$ to the curve $\bar{X}$. Also, we use the computation of multiplicities made above. If $g=e$, then we have the formula $(2 g(X)-2)=[L: K](2 g(C)-2)+$ (local terms) for the behavior of the genus under a finite covering and base changes of the ground field [T2]. For example, if our extension is only weakly ramified (that is $G_{i}=\{e\}$ for $i>1$ ), then we have the Hurwitz formula

$$
(2 g(X)-2)=[L: K](2 g(C)-2)+\sum_{x \in C}\left(e_{x}-1\right),
$$

where $e_{x}$ is the ramification index over the point $x$.

Basically, this result was found by Andre Weil. Here, we have followed Serre's exposition in $[\mathrm{S} 2]$.

4.3. Relationship with explicit formulas. Now we want to combine the explicit formula of Subsection 3.4 and the trace considerations we have just discussed. First, we review the explicit formulas, using Weil's exposition W2. Again, we deal with an algebraic curve $C$, the field $K=\mathbb{F}_{q}(C)$, and its maximal Abelian extension $K^{a b}$. By class field theory, we have the reciprocity map $\mathbb{A}_{C}^{*} / K^{*} \rightarrow \operatorname{Gal}\left(K^{a b} / K\right)$ and, also, the degree map deg : $\mathbb{A}_{C}^{*} / K^{*} \rightarrow \mathbb{Z}$. We start with a function $f=f(n), n \in \mathbb{Z}$, where the group $\mathbb{Z}$ is the target of the degree map. Assume that $f \in \mathcal{D}(\mathbb{Z})$, so that its Laurent transform $\tilde{f}$ belongs to $\mathbb{C}\left[\mathbb{C}^{*}\right]$. With Weil, we introduce the functional

$$
S(f)=\sum_{P(z)=0} \tilde{f}(z),
$$

where $P$ is the numerator of the zeta function of $C$. The degree map allows us to regard $f$ as a function on the group $\mathbb{A}_{C}^{*} / K^{*}$. Also, since we have embeddings $K_{x}^{*} \subset \mathbb{A}_{C}^{*} / K^{*}$, we can restrict $f$ to $K_{x}^{*}$ and get a function $f_{x}$ for each point $x \in C$. We have the following 
distributions:

$$
\begin{aligned}
D_{x}(f) & =\int_{\mathcal{O}_{x}^{*}} \frac{f_{x}(g)-f_{x}(1)}{|1-g|} d^{*} g+\int_{K_{x}^{*}-\mathcal{O}_{x}^{*}} \frac{f_{x}(g)}{|1-g|} d^{*} g, \\
D(f) & =\int_{\mathbb{A}_{C}^{*} / K^{*}} f(g)\left[|g|^{1 / 2}+|g|^{-1 / 2}\right] d^{*} g .
\end{aligned}
$$

In our setting, the Weil explicit formula reads

$$
S(f)=(2-2 g(C)) \delta_{e}(f)+D(f)-\sum_{x \in C} D_{x}(f) .
$$

By definition, we have $D_{x}(f)=a_{x}\left(f_{x}\right)$; therefore, we can compare the expressions (39) and (38). For this, we need to rewrite (38) for functions of the elements $g \in G$.We see that $S(f)-D(f)$ will correspond to the Lefschetz trace in cohomology. The first term $S(f)$ corresponds to the trace on the group $H^{1}\left(X, \mathbb{Q}_{l}\right)$, and the second term $D(f)$ to the traces on the groups $H^{0}\left(\bar{X}, \mathbb{Q}_{l}\right)$ and $H^{2}\left(\bar{X}, \mathbb{Q}_{l}\right)$.

The papers [C, M, G] contain an interpretation of all terms in the Weil formula as traces. Connes used representations in Hilbert spaces, Meyer in Bruhat-Schwartz spaces. Here, we give our own, much more elementary, version.

Consider the Lefschetz formula for integral powers of the Frobenius map. For any $n \geq 1$, we have (by (36)):

$$
\begin{aligned}
\# C\left(\mathbb{F}_{q^{n}}\right) & =\# \bar{C}^{F r^{n}}=\operatorname{Tr}\left(\left(F r^{*}\right)^{n} \mid H^{\bullet}\left(\bar{C}, \mathbb{Q}_{l}\right)\right), \\
2-2 g(C) & =\operatorname{Tr}\left(\left(F r^{*}\right)^{0} \mid H^{\bullet}\left(\bar{C}, \mathbb{Q}_{l}\right)\right), \\
q^{-n} \# C\left(\mathbb{F}_{q^{n}}\right) & =q^{-n} \# \bar{C}^{F r^{n}}=\operatorname{Tr}\left(\left(F r^{*}\right)^{-n} \mid H^{\bullet}\left(\bar{C}, \mathbb{Q}_{l}\right)\right) .
\end{aligned}
$$

Let $f: \mathbb{Z} \rightarrow \mathbb{C}$ be a function on $\mathbb{Z}$ with finite support. Recall that, by class field theory, $1 \in \mathbb{Z}=\mathbb{A}^{*} / \mathbb{A}^{*(1)}$ corresponds to the Frobenius automorphism of the field $\overline{\mathbb{F}_{q}}$ over $\mathbb{F}_{q}$. Thus, we may introduce

$$
f(F r):=\sum_{n \in \mathbb{Z}} f(n) F r^{-n}
$$

and conclude that

$\sum_{n \geq 1} f(n) q^{-n} \# C\left(\mathbb{F}_{q^{n}}\right)+(2-2 g(C)) f(0)+\sum_{n \leq-1} f(n) \# C\left(\mathbb{F}_{q^{-n}}\right)=\operatorname{Tr}\left(f(F r) \mid H^{\bullet}\left(\bar{C}, \mathbb{Q}_{l}\right)\right)$.

As usual, we denote by $\tilde{f}(z)=\sum_{n} f(n) z^{n}$ the Laurent transform of the function $f$. The right-hand side of the equation is equal to

$$
\tilde{f}(1)+\tilde{f}(q)-\sum_{P(z)=0} \tilde{f}(z) .
$$

On the left-hand side, we get

$$
\begin{aligned}
& \sum_{n \geq 1}\left(\sum_{x \in C\left(\mathbb{F}_{q^{n}}\right)} 1\right) q^{-n} f(n)+(2-2 g(C)) f(0)+\sum_{n \leq-1}\left(\sum_{x \in C\left(\mathbb{F}_{q}-n\right)} 1\right) f(n) \\
& =(2-2 g(C)) f(0)+\sum_{n \geq 1} \sum_{\substack{x \in C, \operatorname{deg}(x) \mid n}} \operatorname{deg}(x) q^{-n} f(n)+\sum_{n \geq 1} \sum_{\substack{x \in C, \operatorname{deg}(x) \mid n}} \operatorname{deg}(x) f(-n) \\
& =(2-2 g(C)) f(0)+\sum_{\substack{n \geq 1, x \in C}} \operatorname{deg}(x) q^{-n \operatorname{deg}(x)} f(n \operatorname{deg}(x))+\sum_{\substack{n \geq 1, x \in C}} \operatorname{deg}(x) f-(n \operatorname{deg}(x)) .
\end{aligned}
$$


Here, we have used the fact that, in accordance with (34), we have $\# \bar{C}^{F r^{n}}=\# C\left(\overline{\mathbb{F}}_{q^{n}}\right)=$ $\sum_{x \in C, \operatorname{deg}(x) \mid n} \operatorname{deg}(x)$. Once again, we arrive at the same explicit formula (26) that was explained above.

\section{$\S 5$. Number Fields (From $\mathbb{C}^{*}$ TO $\left.\mathbb{C}\right)$}

It is certainly interesting to understand what corresponds to the constructions developed above in the case of number fields. A fundamental analogy between number fields $(=$ finite extensions of $\mathbb{Q})$ and fields of algebraic functions of one variable over a finite field (= fields of functions on algebraic curves) (see, e.g., [P2]) shows that we can expect that there are some similar constructions. Actually, the adelic approach to the zeta- and $L$-functions developed by Tate and Iwasawa has realized this idea with respect to the analytic properties of these functions (see especially the unified exposition by Weil. [W1]21) Thus, what was said in $\S 2$ can be transferred to the number field situation with appropriate modifications 22 The main problem is to deal with the Archimedean valuations of the fields.

If we wish to reconsider our duality constructions for number fields, then first we must note that there is no substitution $t=q^{-s}$ for the zeta-function because we no longer have any $q$. The $L$-functions are very simple analytic functions in the case of curves, being superpositions of a rational function and the exponential function. This is already no longer true for the Riemann zeta-function. This means that we need to reformulate our results on the $s$-plane, forgetting the variables such as $z$.

The map $s \mapsto z=q^{-s}$ is a universal covering of the torus $\mathbb{C}^{*}$ under an action of the discrete group $\mathbb{Z}$, and the $s$-plane can be viewed as $\operatorname{Hom}(\mathbb{Z}, \mathbb{C})$ if we start with the group $\mathbb{Z}$. The action is generated by the map $s \mapsto s+2 \pi i / \ln q$. We shall discuss this issue in more detail later in the text; now we simply rewrite our residue sum on the $s$-plane.

Let $f=\delta_{(\geq 0)} \in \mathcal{D}_{+}(\Gamma)$ for the group $\Gamma_{C}$ associated with a curve $C$. Then $\tilde{f}=Z_{C}(z)$. If we rewrite the sum of residues for $\tilde{f} \omega_{0}$ on the $s$-plane we meet the problem that there are infinitely many poles lying on arithmetical progressions. The easiest way to overcome this difficulty is to consider a fundamental domain for the group $\mathbb{Z}$. We may take a horizontal strip $\pi / \ln q \geq t \geq-\pi / \ln q$. The image of this strip will be exactly the open subset $\mathbb{C}^{*}$ of $\mathbb{P}^{1}$. We need to add two infinite points $\pm \infty$ to the strip, and then

$$
\operatorname{res}_{(+\infty)} \zeta_{C}(s) d s+\operatorname{res}_{(0)} \zeta_{C}(s) d s+\operatorname{res}_{(1)} \zeta_{C}(s) d s+\operatorname{res}_{(-\infty)} \zeta_{C}(s) d s=0 .
$$

In the "open" strip, there are only two poles, and we can observe that the integral over the rectangle $\gamma$ with edges $\gamma_{1}, \gamma_{2}, \gamma_{3}, \gamma_{4}$, where

$$
\begin{aligned}
\gamma_{1} & =\{A+i t:-\pi / \ln q \leq t \leq \pi / \ln q\}, \\
\gamma_{2} & =\{\sigma+i T:-A \leq \sigma \leq A\}, \\
\gamma_{3} & =\{-A+i t:-\pi / \ln q \leq t \leq \pi / \ln q\}, \\
\gamma_{4} & =\{\sigma+i T:-A \leq \sigma \leq A\},
\end{aligned}
$$

$s=\sigma+i t$, and $A$ is sufficiently large, is zero. Of course, the integrals over the vertical boundaries of the strip do not depend on the choice of $A$. Thus, the sum makes sense without any additional infinite points and, certainly, the sum defined as this integral is equal to our previous sum of residues on the $z$-torus. This suggests what to do in the case of a number field $K$. Denote by $\xi_{K}(s)$ the "complete" zeta-function of the field $K$. This is

\footnotetext{
${ }^{21}$ At the same time, I. R. Shafarevich gave a course of lectures at Moscow University where a unified exposition was also presented $[\mathrm{Sh}$. An interesting historical coincidence!

${ }^{22}$ Historically, the passage was in the opposite direction!
} 
Dedekind's zeta function completed by the gamma-factors attached to the Archimedean places of the field $K$ (see the Introduction for the case of $K=\mathbb{Q}$, the Appendix for the general case, and [W2, [L1]).

We wish to examine the sum

$$
\operatorname{res}_{(0)} \xi_{K}(s) d s+\operatorname{res}_{(1)} \xi_{K}(s) d s-\lim _{A \rightarrow+\infty} \int_{A-i \infty}^{A+i \infty} \xi_{K}(s) d s+\lim _{A \rightarrow-\infty} \int_{A-i \infty}^{A+i \infty} \xi_{K}(s) d s
$$

and to show that it is equal to zero 23 This is actually the case (see Appendix) and we see that our observation also has some meaning for number fields. Now we describe what should stand for function spaces and maps from $\S 3$ in the number field case.

Let $K / \mathbb{Q}$ be a finite extension of $\mathbb{Q}$ and $\mathbb{A}_{K}$ the adelic group (= adelic product of all local fields $K_{v}$ for all places $v$ ). We set

$$
\begin{aligned}
& K_{v}^{*} \supset \widehat{\mathcal{O}}_{v}^{*} \text { for finite (non-Archimedean) places } v \\
& K_{v}^{*} \supset \widehat{\mathcal{O}}_{v}^{*}:=\{ \pm 1\} \quad \text { if } \quad K_{v}=\mathbb{R} \\
& K_{v}^{*} \supset \widehat{\mathcal{O}}_{v}^{*}:=\{|z|=1\} \quad \text { if } \quad K_{v}=\mathbb{C}
\end{aligned}
$$

and $\widehat{\mathcal{O}}^{*}=\prod_{v} \widehat{\mathcal{O}}_{v}^{*}$. This is the maximal compact subgroup in $\mathbb{A}_{K}^{*}$. We may take

$$
\begin{aligned}
\Gamma_{K} & =\bigoplus_{v} K_{v}^{*} / \widehat{\mathcal{O}}_{v}^{*}=\bigoplus_{\text {finite } v} \mathbb{Z} \bigoplus_{\text {infinite } v} \mathbb{R} \\
& =(\text { discrete group of ideals }) \oplus(\text { vector space over } \mathbb{R}) .
\end{aligned}
$$

Now we need to define a space $\mathcal{S}\left(\Gamma_{K}\right)$ and a map

$$
\mathcal{S}\left(\mathbb{A}_{K}\right)^{\hat{\mathcal{O}}^{*}} \stackrel{\sim}{\rightarrow} \mathcal{S}\left(\Gamma_{K}\right) .
$$

The space $\mathcal{S}\left(\mathbb{A}_{K}\right)$ of Bruhat-Schwartz functions is well defined in $\mathrm{Br}$. (see also $\mathrm{Sch}$, OP2 ). On the local Archimedean components $K_{v}$ of $\mathbb{A}_{K}$, these spaces are the Schwartz spaces, and on the local non-Archimedean components $K_{v}$ of $\mathbb{A}_{K}$, they are the Bruhat spaces $\mathcal{D}\left(K_{v}\right)$ (see the beginning of the Introduction). For the local spaces $\mathcal{S}\left(\Gamma_{v}\right)$, we may choose the spaces $\mathcal{S}\left(K_{v}\right)^{\hat{\mathcal{O}}_{v}^{*}}$ themselves, viewing them as function spaces on $\Gamma_{v}$.

In the case of finite places, the groups $\Gamma_{v}$ are equal to $\mathbb{Z}$ and we can develop the theory exactly as in the case of algebraic curves. Namely, we can introduce a holomorphic torus $\mathbb{T}_{v}$ and the same function spaces $\mathcal{D}\left(\Gamma_{v}\right) \cong \mathbb{C}\left[\mathbb{T}_{v}\right]$ and $\mathcal{D}_{+}\left(\Gamma_{v}\right) \cong \mathbb{C}_{+}\left[\mathbb{T}_{v}\right]$ as above. The Fourier transform will be well defined in the same way, but the numbers $q_{v}\left(=q_{x}\right)$ are not the powers of a single $q$. For example, if $K=\mathbb{Q}$, then the $v$ correspond to all primes $p$ and $q_{v}=p$. For this reason and (more importantly) by the structure of Archimedean places, we are forced to consider function spaces on the groups $\overline{\mathbb{T}}_{v}$, universal coverings of the tori $\mathbb{T}_{v}$. Surely, $\mathbb{T}_{v} \cong \mathbb{C}$. For simplicity, now we consider the case where $K=\mathbb{Q}$. 24

Then, instead of the space $\mathcal{D}\left(\mathbb{Q}_{p}\right)^{\mathbb{Z}_{p}^{*}}$, we have the following space on $\mathbb{C}$ :

$$
\mathcal{F}_{p}(\mathbb{C}):=\left\{P\left(p^{-s}\right) \mid P \in \text { the space of Laurent polynomials, } s \in \mathbb{C}\right\},
$$

and the space $\mathcal{D}_{+}\left(\mathbb{Q}_{p}\right)^{\mathcal{O}_{p}^{*}}$ is replaced with the space

$$
\mathcal{F}_{p,+}(\mathbb{C}):=\left\{\left(1-p^{-s}\right)^{-1} P\left(p^{-s}\right) \mid P \in \text { the space of Laurent polynomials, } s \in \mathbb{C}\right\} .
$$

A more complicated problem is what to choose for the infinite, Archimedean places. In our case, $K_{v}=\mathbb{R}$. The largest possible choice is the space $\mathcal{S}(\mathbb{R})$. The traditional choice

\footnotetext{
${ }^{23}$ This question was discussed during my lectures in Moscow. The ideas on how to deal with this sum belong to Volodya Zhgun and Irina Rezvyakova.

${ }^{24}$ The interested reader can easily extend this discussion to a general number field setting, using, e.g., W2 Chapter VII, nos. 2, 6], L1, Chapter VII, no. 4].
} 
will be the much smaller subspace

$$
\mathcal{D}_{+}(\mathbb{R})=\left\{\text { the linear span of } x^{n} \exp \left(-a x^{2}\right), x \in \mathbb{R}, n \in \mathbb{Z}_{\geq 0}, a \in \mathbb{R}_{+}\right\},
$$

and for the space of invariants under $\widehat{\mathcal{O}}_{v}^{*}=\{ \pm\}$ we restrict ourselves to the even powers of $x$ 's 25

The Fourier transform on the group $\mathbb{R}$ (see the Introduction) preserves the space $\mathcal{D}_{+}(\mathbb{R})$. Indeed, the function $\exp \left(-\pi x^{2}\right)$ is invariant under the Fourier transform, so that we get

$$
\mathrm{F}\left(\exp \left(-\pi a x^{2}\right)\right)=\frac{1}{\sqrt{a}} \exp \left(-\pi a^{-1} x^{2}\right)
$$

From the point of view of analogy between the fields such as $\mathbb{Q}_{p}, \mathbb{F}_{p}((t))$ and $\mathbb{R}$, it is worth comparing this formula with the action of the Fourier transform on the standard functions from Subsection 3.1: $\mathrm{F}\left(\delta_{\geq m}\right)=q^{-1 / 2 k-m} \delta_{\geq-k-m}$ with $k=0$.

More generally, for a polynomial $P(x)$ we have

$$
\mathrm{F}\left(P(x) \exp \left(-\pi a x^{2}\right)\right)=Q(x) \exp \left(-\pi a^{-1} x^{2}\right)
$$

with a new polynomial $Q(x)$. See the details in [W2, Chapter VII, no. 2, Proposition 3].

Now, we use the classical Mellin transform M:

$$
\mathrm{M}_{v}(f)=\int_{\mathbb{R}_{+}^{*}}|x|^{s} f(x) \frac{d x}{|x|}, \quad x \in K_{v}, \quad f \in \mathcal{D}_{+}\left(K_{v}\right) .
$$

Using the standard integral presentation for the $\Gamma$-function, we see that, for the field $K_{v}=\mathbb{R}, v=\infty$,

$$
\mathrm{M}_{\infty}\left(x^{2 n} \exp \left(-a x^{2}\right)\right)=a^{-n} a^{-s / 2} \Gamma(s / 2+n) .
$$

This suggests the following definition:

$$
\mathcal{F}_{\infty,+}(\mathbb{C}):=\left\{\text { the linear span of } a^{-s / 2} \Gamma(s / 2+n), s \in \mathbb{C}, n \in \mathbb{Z}_{\geq 0}, a \in \mathbb{R}_{+}\right\} 26
$$

Now we proceed along the line developed for the case of algebraic curves. We introduce the whole space $\bigotimes_{v} \mathcal{F}_{v,+}(\mathbb{C})$; then we map a single copy of $\mathbb{C}$ into the product of the $\mathbb{C}$ 's over all places $v$ and, by restriction, we get a space $\mathcal{F}_{+}(\mathbb{C})$. Then, the functional equation for the zeta-function of the field $K$ (see [L1, W2]) should imply the commutativity of the diagram

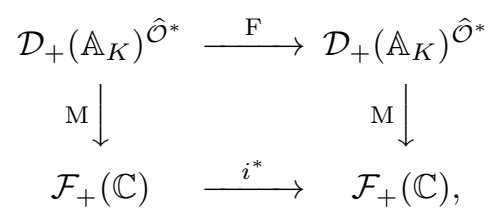

where the global Mellin map $\mathrm{M}$ is a product of the local ones $\mathrm{M}_{v}$ over all $v$, and $i: \mathbb{C} \rightarrow \mathbb{C}$ with $i(s)=1-s$. It can also be shown that the Poisson formula becomes the residue relation on the $s$-plane we have stated above. Nevertheless, we want to finish with the following question.

Question. Does there exist an analog of the group dual to the group $\Gamma_{K}$ for a number field $K$, and how can one realize the whole analysis working entirely on this group?

\footnotetext{
${ }^{25}$ Sergey Gorchinskiu has asked what an analog of the space $\mathcal{D}\left(\mathbb{Q}_{p}\right)$ is. A good question!

${ }^{26}$ In classical analysis, the choice of smaller subspaces in $\mathcal{S}(\mathbb{R})$, with stronger conditions for growth at infinity, implies better analytic properties of the Mellin transform (the so-called Paley-Wiener type

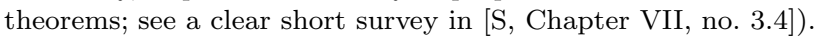




\section{$\S 6$. Appendix (Irina Rezvyakova). Residues of the Dedekind zeta functions OF NUMBER FIELDS}

Let $\mathbb{Q}$ be the field of rational numbers, $K \supset \mathbb{Q}$ its finite extension of degree $d=r_{1}+2 r_{2}$, and $D$ its discriminant.

The Dedekind zeta function of the field $K$ can be defined by the following formula for $\operatorname{Re}(s)>1$ :

$$
\zeta_{K}(s)=\sum_{\mathfrak{a}} \mathrm{Nm}(\mathfrak{a})^{-s}=\sum_{n=1}^{+\infty} \frac{a(n)}{n^{s}},
$$

where

$$
a(n)=\sum_{\mathfrak{a}: \operatorname{Nm}(\mathfrak{a})=n} 1 .
$$

Here, summation in the first identity is over all nonzero integral ideals $\mathfrak{a}$ in the ring of integers of the field $K$, and $\operatorname{Nm}(\mathfrak{a})$ denotes the norm of the ideal $\mathfrak{a}$.

Starting with $\zeta_{K}(s)$, one can define the $\xi$-function

$$
\xi_{K}(s)=G_{1}(s)^{r_{1}} G_{2}(s)^{r_{2}} \zeta_{K}(s),
$$

where

$$
G_{1}(s)=\pi^{-s / 2} \Gamma(s / 2), \quad G_{2}(s)=(2 \pi)^{1-s} \Gamma(s) .
$$

(compare with the corresponding extension of Riemann's zeta-function in $\S 1$ ). This function is meromorphic on the entire complex plane with first order poles at $s=0$ and $s=1$. It satisfies the following functional equation L1, W2 :

$$
\xi_{K}(s)=|D|^{\frac{1}{2}-s} \xi_{K}(1-s) .
$$

Let $A>1$ and $T$ be sufficiently large real numbers. Consider the rectangle $\Gamma=$ $\gamma_{1} \cup \gamma_{2} \cup \gamma_{3} \cup \gamma_{4}$ :

$$
\begin{aligned}
& \gamma_{1}=\{A+i t:-T \leq t \leq T\}, \\
& \gamma_{2}=\{\sigma+i T: 1-A \leq \sigma \leq A\}, \\
& \gamma_{3}=\{1-A+i t:-T \leq t \leq T\}, \\
& \gamma_{4}=\{\sigma+i T: 1-A \leq \sigma \leq A\},
\end{aligned}
$$

where $s=\sigma+i t$. By Cauchy's theorem, we get

$$
\frac{1}{2 \pi i} \int_{\Gamma} \xi_{K}(s) d s=\underset{s=0}{\operatorname{res}} \xi_{K}(s)+\underset{s=1}{\operatorname{res}} \xi_{K}(s) .
$$

We want to show that if $A$ is fixed and $T \rightarrow+\infty$, then the integrals over the paths $\gamma_{2}, \gamma_{4}$ tend to zero. This can be done in four steps.

1) The Stirling formula for the gamma function implies that in the domain $|\operatorname{Re}(s)|$ $\leq a,|\operatorname{Im}(s)| \geq 1(s=\sigma+i t)$ we have

$$
|\Gamma(s)| \ll|t|^{\sigma-\frac{1}{2}} e^{-\frac{\pi}{2} t}
$$

(see, e.g., [VK, Appendix, §3]).

2) In the right half-plane, under the condition $\operatorname{Re}(s) \geq 1+\varepsilon$ (where $\varepsilon$ is an arbitrarily small positive number), we have the estimate

$$
\left|\zeta_{K}(s)\right| \leq c=c(\varepsilon)
$$


Proof. Looking at the Dirichlet series $\zeta_{K}(s)=\sum_{\mathfrak{a}} \mathrm{Nm}(\mathfrak{a})^{-s}$, we can rewrite it as

$$
\sum_{\substack{n \geq 1, \mathfrak{a} \cap \mathbb{Z}=(n) \\ n \in \mathbb{Z}}} \operatorname{Nm}(\mathfrak{a})^{-s}
$$

This immediately reduces the problem to the corresponding estimate for Riemann's zeta function, where it is obvious.

3) From 1), 2) and the functional equation we see that the inequality

$$
\left|\zeta_{K}(s)\right| \leq c_{1}(\varepsilon)|t|^{d\left(\frac{1}{2}-\sigma\right)}
$$

is valid for $-a \leq \operatorname{Re}(s) \leq-\varepsilon,|\operatorname{Im}(s)| \geq 1$.

4) To get a bound in the critical strip, we apply the Phragmen-Lindelöf principle.

First, to find the order of growth of Dedekind's zeta function in the domain $-\varepsilon \leq$ $\operatorname{Re} s \leq 1+\varepsilon$, we use the Phragmen-Lindelöf principle in the following form. Let $a$ function $f$ be holomorphic in a neighborhood of the strip $a \leq \operatorname{Re} s \leq b$ where the estimate $|f(s)| \ll e^{|s|^{A}}$ is fulfilled with some $A \geq 0$. If

$$
\begin{gathered}
|f(a+i t)| \leq c_{a}(1+|t|)^{\alpha}, \\
|f(b+i t)| \leq c_{b}(1+|t|)^{\beta},
\end{gathered}
$$

then for $a \leq \sigma \leq b$, we have

$$
|f(\sigma+i t)| \leq c_{a}^{l(\sigma)} c_{b}^{1-l(\sigma)}(1+|t|)^{\alpha l(\sigma)+\beta(1-l(\sigma))},
$$

where $l(\sigma)$ is a linear function such that $l(a)=1, l(b)=0$.

We apply this statement to the holomorphic function $f(s)=(s-1) \zeta_{K}(s)$ in the strip with ends $a=-\varepsilon, b=1+\varepsilon$, with parameters $\alpha=d(1 / 2+\varepsilon)+1, \beta=1$, and with the function $l(\sigma)=-\frac{1}{1+2 \varepsilon} \sigma+\frac{1+\varepsilon}{1+2 \varepsilon}$. Using the principle, we see that in the given strip

$$
|f(\sigma+i t)| \ll_{\varepsilon}(1+|t|)^{1+d(1 / 2+\varepsilon)\left(-\frac{1}{1+2 \varepsilon} \sigma+\frac{1+\varepsilon}{1+2 \varepsilon}\right)} \ll_{\varepsilon}(1+|t|)^{1+d\left(-\frac{\sigma}{2}+\frac{1+\varepsilon}{2}\right)} .
$$

Therefore, for $-\varepsilon \leq \sigma \leq 1+\varepsilon,|t| \geq 1$ we have

$$
\left|\zeta_{K}(\sigma+i t)\right| \ll_{\varepsilon}|t|^{d\left(\frac{1-\sigma}{2}+\frac{\varepsilon}{2}\right)} .
$$

Thus, in the domain $\operatorname{Re}(s) \geq-a,|t| \geq 1$, we obtain

$$
\left|\zeta_{K}(\sigma+i t)\right| \ll|t|^{d\left(\frac{1+a}{2}\right)} .
$$

$5)$ So, the integrals of the function $\xi_{K}(s)$ over the paths $\gamma_{2}, \gamma_{4}$ can be bounded from above by a constant depending only on $A$ :

$$
T^{A-\frac{1}{2}} e^{-\frac{\pi}{2} T} T^{d\left(\frac{1}{2}+A\right)} \ll e^{-\frac{\pi}{2} T} T^{(d+1)\left(A+\frac{1}{2}\right)} \rightarrow 0 \quad \text { as } \quad T \rightarrow+\infty .
$$

Consequently, passing to the limit as $T \rightarrow+\infty$, we get the relation

$$
\frac{1}{2 \pi i} \int_{(A)} \xi_{K}(s) d s-\frac{1}{2 \pi i} \int_{(1-A)} \xi_{K}(s) d s=\underset{s=0}{\operatorname{res}} \xi_{K}(s)+\underset{s=1}{\operatorname{res}} \xi_{K}(s),
$$

where $(A)$ denotes the line $\operatorname{Re}(s)=A$.

Example. Let $K=\mathbb{Q}(\sqrt{-D})$ be an imaginary quadratic field, where $-D \leq-3$. Denote by $h=h(-D)$ the class number of the field $K$ and by $\omega$ the number of units (recall that $\omega=2$ when $D>4)$. In this case, the $\xi$-function is equal to

$$
\xi_{K}(s)=(2 \pi)^{-s} \Gamma(s) \zeta_{K}(s)
$$

and satisfies

$$
\xi_{K}(s)=D^{\frac{1}{2}-s} \xi_{K}(1-s) .
$$


We find the residues:

$$
\operatorname{res}_{s=1} \xi_{K}(s)=\frac{1}{2 \pi} \underset{s=1}{\operatorname{res}} \zeta_{K}(s)=\frac{h}{\omega \sqrt{D}} .
$$

The functional equation implies

$$
\underset{s=0}{\operatorname{res}} \xi_{K}(s)=\zeta_{K}(0)=\left(\frac{2 \pi}{\sqrt{D}}\right)^{-1}\left(-\underset{s=1}{\operatorname{res}} \zeta_{K}(s)\right)=-\frac{h}{\omega} .
$$

Now, we evaluate the integrals. Applying the functional equation and then the Mellin transform, we come to the formula

$$
\begin{aligned}
\frac{1}{2 \pi i} \int_{(1-A)} \xi_{K}(s) d s & =\frac{1}{2 \pi i} \int_{(1-A)} D^{\frac{1}{2}-s} \xi_{K}(1-s) d s=\frac{1}{2 \pi i} \int_{(A)} D^{s-\frac{1}{2}} \xi_{K}(s) d s \\
& =D^{-\frac{1}{2}} \sum_{n=1}^{+\infty} a(n) \frac{1}{2 \pi i} \int_{(A)} \Gamma(s)\left(\frac{2 \pi n}{D}\right)^{-s} d s \\
& =D^{-\frac{1}{2}} \sum_{n=1}^{+\infty} a(n) e^{-\frac{2 \pi n}{D}}=D^{-\frac{1}{2}} \sum_{\mathfrak{a}} e^{-\frac{2 \pi N m(\mathfrak{a})}{D}}
\end{aligned}
$$

Also,

$$
\frac{1}{2 \pi i} \int_{(A)} \xi_{K}(s) d s=\sum_{n=1}^{+\infty} a(n) e^{-2 \pi n}=\sum_{\mathfrak{a}} e^{-2 \pi \mathrm{Nm}(\mathfrak{a})} .
$$

We see that

$$
\frac{h}{\omega}+\sum_{\mathfrak{a}} e^{-2 \pi N m(\mathfrak{a})}=\frac{h}{\omega \sqrt{D}}+D^{-\frac{1}{2}} \sum_{\mathfrak{a}} e^{-\frac{2 \pi N m(\mathfrak{a})}{D}} .
$$

This identity also follows from the functional equation for the corresponding theta-series. Namely, if $\mathcal{A}$ is a class of ideals, then the function

$$
\theta_{\mathcal{A}}(z)=\frac{1}{\omega}+\sum_{\mathfrak{a} \in \mathcal{A}} e^{2 \pi i z \operatorname{Nm}(\mathfrak{a})}, \quad \operatorname{Im} z>0,
$$

satisfies the functional equation

$$
\theta_{\mathcal{A}}(z)=\frac{i}{z \sqrt{D}} \theta_{\mathcal{A}^{-1}}\left(-\frac{1}{D z}\right)
$$

(see, e.g., [H2]). Thus, the function $\theta_{K}(z)=\sum_{\mathcal{A}} \theta_{\mathcal{A}}(z)=\frac{h}{\omega}+\sum_{\mathfrak{a}} e^{2 \pi i z \mathrm{Nm}(\mathfrak{a})}$ satisfies a similar functional equation

$$
\theta_{K}(z)=\frac{i}{z \sqrt{D}} \theta_{K}\left(-\frac{1}{D z}\right)
$$

Taken at the point $z=i$, this relation turns exactly into the identity that we obtained from the Cauchy formula.

\section{REFERENCES}

[A] J. W. S. Cassels and A. Fröhlich (eds.), Algebraic number theory, Acad. Press, London-New York, 1967. MR0215665(35:6500)

[Br] F. Bruhat, Distributions sur un groupe localement compact et applications à l'étude des représentations des groupes $\mathcal{W}$-adiques, Bull. Soc. Math. France 89 (1961), 43-75. MR0140941 $(25: 4354)$

[C] A. Connes, Trace formulas in noncommutative geometry and the zeros of the Riemann zeta function, Preprint IHES/M/98/72, 1998, 88 pp.

[Den] C. Deninger, Lefschetz trace formulas and explicit formulas in analytic number theory, J. Reine Angew. Math. 441 (1993), 1-15. MR.1228608 (85d:11110) 
[D] M. Deuring, Lectures on the theory of algebraic functions of one variable, Lecture Notes in Math., vol. 314, Springer-Verlag, Berlin-New York, 1973. MR0344231 (49:8970)

[Dix] J. Dixmier, Les $C^{*}$-algèbres et leurs représentations, Cahiers Sci., fasc. 29, Gauthier-Villars, Paris, 1969. MR0246136(39:7442)

[GSh] I. M. Gel'fand and G. E. Shilov, Generalized functions. Vyp. 1. Generalized functions and operations on them, Fizmatgiz, Moscow, 1959; English transl., Generalized functions. Vol. 1. Properties and operations, Acad. Press, New York-London, 1964 [1977]. MR0435831 (55:8786a)

[GGPSh] I. M. Gel'fand, M. I. Graev, and I. I. Pyatetskiu-Shapiro, Generalized functions. Vyp. 6. Theory of representations and automorphic functions, Nauka, Moscow, 1966; English transl., Representation theory and automorphic functions, W. B. Saunders Co., Philadelphia, 1969. MR.0220673 (36:3725) MR0233772 (38:2093)

[G] D. Goldfeld, A spectral interpretation of Weil's explicit formula, Lecture Notes in Math., vol. 1593, Springer-Verlag, Berlin, 1994, pp. 135-152. MR1329730 (96f:11110)

[H1] E. Hecke, Über die Zetafunktion beliebiger algebraischer Zahlkorper, Gött. Nachr. (1917), 7789.

[H2] _ Vorlesungen über die Theorie der algebraischen Zahlen, Akad. Verlagsges, Leipzig, 1923.

[I1] K. Iwasawa, A note on functions, Proc. of the Internat. Congress of Mathematicians (Cambridge, Mass., 1950). Vol. 2, Amer. Math. Soc., Providence, RI, 1952, p. 322.

[I2] Letter to J. Dieudonné, Zeta Functions in Geometry (Tokyo, 1990), Adv. Stud. Pure Math., vol. 21, Kinokuniya, Tokyo, 1992, pp. 445-450. MR1210798 (94f:11118)

[L1] S. Lang, Algebraic numbers, Addison-Wesley, Reading, 1964. MR0160763 (28:3974)

[L2] _ Algebraic number theory, Addison-Wesley, Reading, 1970. MR0282947 (44:181)

[M] R. Meyer, On a representation of the idele class group related to primes and zeros of L-functions, Duke Math. J. 127 (2005), 519-595. MR2132868(2006e:11128)

[OP1] D. V. Osipov and A. N. Parshin, Harmonic analysis on local fields and adelic spaces. I, Izv. Ross. Akad. Nauk Ser. Mat. 72 (2008), no. 5, 77-140; English transl., Izv. Math. 72 (2008), no. 5, 915-976; e-print arXiv:0707.1766. MR2473773 (2010a:11225)

[OP2] Harmonic analysis on local fields and adelic spaces. II, Izv. Ross. Akad. Nauk Ser. Mat. 75 (2011), no. 4, 91-164; English transl., Izv. Math. 75 (2011), 749-814. e-print arXiv:0912.1577. MR2866188

[OP3] Harmonic analysis and the Riemann-Roch theorem, e-print arXov: 1107.0408.

[P1] A. N. Parshin, Harmonic analysis on adelic spaces and local fields, Mathematisches Forschungsinstitut Oberwolfach, Report No. 43/2005, Arakelov Geometry, September 2005, pp. 2471-2474.

[P2] Numbers as functions: the development of an idea in the Moscow school of algebraic geometry, Mathematical Events of XX Century (A. Bolibrukh et al., eds.), Fazis, Moscow, 2003, pp. 363-397; e-print arXiv: 0912.3785. (Russian)

[P3] - On holomorphic representations of discrete Heisenberg groups, Funktsional. Anal. i Prilozhen. 44 (2010), no. 2, 92-96; English transl., Funct. Anal. Appl. 44 (2010), no. 2, 156-159. MR2681962

[P4] , Representations of higher adelic groups and arithmetic, Proc. ICM, Vol. 1, Hindustan Book Agency, New Delhi, 2010, pp. 362-392; e-print arXiv: 1012.0486. MR2827898

[P5] _ Lectures on representations of discrete Heisenberg groups, Humboldt Univ., Berlin, October 2010 (Notes by Aaron Greicius and Hartwig Mayer).

[R] B. Riemann, Über die Anzahl der Primzahlen unter einer gegebenen Grösse, Monatsb. der Berliner Akad. 1858/1860, 671-680 (Gesamm. Math. Werke, Teubner, Leipzig, 1982, No. VII, S. $145-155)$.

[Sch] L. Schwartz, Théorie des distributions, Tome II. Actualités Sci. Indust., no. 1122, Hermann, Paris, 1951. MR0041945 (12:833d)

[Sh] I. R. Shafarevich, The zeta-function. Notes of lectures 1966-1967, Moskov. Gos. Univ., Moscow, 1969. (Russian)

[S] G. E. Shilov, Mathematical analysis: Special course, Fizmatgiz, Moscow, 1961. (Russian) MR0131328(24:A1180)

[S1] J.-P. Serre, Groupes algébriques et corps de classes, Publ. Inst. Math. Univ. Nancago, No. 7, Hermann, Paris, 1959. MR0103191 (21:1973)

[S2] _ Sur la rationalité des représentations d'Artin, Ann. of Math. (2) 72 (1960), 405-420. $\operatorname{MR} 0171775(30: 2002)$

[S3] Corps locaux, Publ. Univ. Nancago, No. 8, Hermann, Paris, 1968. MR0354618 $(50: 7096)$ 
[T1] J. Tate, Fourier analysis in number fields and Hecke's zeta-function, Thesis, Princeton Univ., Ann Arbor, 1950 (stated in [L1] and publ. in [A]). MR2612222

[T2] Genus change in inseparable extensions of function fields, Proc. Amer. Math. Soc. 3 (1952), 400-406. MR0047631 (13:905b)

[VK] S. M. Voronin and A. A. Karatsuba, The Riemann zeta function, Fizmatlit, Moscow, 1994; English transl., Exp. in Math., vol. 5, Walter de Gruyter and Co., Berlin, 1992. MR,1918212 (2003b:11088) MR:1183467 (93h:11096)

[W1] A. Weil, Fonctions zêta et distributions, Séminaire Bourbaki, vol. 9, Exp. No. 312, Soc. Math. France, Paris, 1995, pp. 523-531. MR1610983

[W2] _ Basic number theory, Grundlehren Math. Wiss., Bd. 144, 3rd ed., Springer-Verlag, Berlin, 1974. MR0427267 (55:302)

[W3] Sur les formules explicites de la théorie des nombres, Izv. Akad. Nauk SSSR Ser. Mat. 36 (1972), no. 1, 3-18; English transl., Math. USSR-Izv. 6 (1972), no. 1, 1-17. MR0379440 $(52: 345)$

Steklov Mathematical Institute, Russian Academy of Sciences, Gubkina St. 8, Moscow 119991, Russia

E-mail address: parshin@mi.ras.ru

Received 7/JUL/2010

Translated by THE AUTHOR 